

University of Pennsylvania ScholarlyCommons

Wharton Pension Research Council Working

Papers

Wharton Pension Research Council

9-1-2012

\title{
Functional Disabilities and Nursing Home Admittance
}

Joelle H. Fong

University of New SouthWales, h.fong@unsw.edu.au

Benedict SK Koh

Singapore Management University, skkoh@smu.edu.sg

Olivia S. Mitchell

The Wharton School, University of Pennsylvania, mitchelo@wharton.upenn.edu

Follow this and additional works at: https://repository.upenn.edu/prc_papers

Part of the Economics Commons

Fong, Joelle H.; Koh, Benedict SK; and Mitchell, Olivia S., "Functional Disabilities and Nursing Home Admittance" (2012). Wharton Pension Research Council Working Papers. 162.

https://repository.upenn.edu/prc_papers/162

This paper is posted at ScholarlyCommons. https://repository.upenn.edu/prc_papers/162

For more information, please contact repository@pobox.upenn.edu. 


\title{
Functional Disabilities and Nursing Home Admittance
}

\begin{abstract}
This paper examines how inability to perform activities of daily living relates to the risk of nursing home admission over older adults' life courses. Using longitudinal data on persons over age 50 from the Health and Retirement Study, we show that aging one year boosts the probability of having two or more disabilities by 9 to 12 percent in a multivariate logistic model. Moreover, at least three-fifths of all 65-year-old men and threequarters of women will experience disability levels during their remaining lifetimes severe enough to trigger nursing home admission. Our analysis also suggests that certain types of disability are more important than others in predicting nursing home admittance and use, which has implications for the design and benefit triggers for long-term care insurance programs.
\end{abstract}

\section{Disciplines}

Economics 


\title{
Functional Disabilities and Nursing Home Admittance
}

\author{
Joelle H. Fong, Benedict SK. Koh, and Olivia S. Mitchell
}

September 2012

\author{
BWP2012-02 \\ Boettner Center Working Paper \\ Boettner Center for Pensions and Retirement Research \\ The Wharton School, University of Pennsylvania \\ 3620 Locust Walk, 3000 SH-DH \\ Philadelphia, PA 19104-6302
}

Tel: 215.898.7620 Fax: 215.573.3418

Email:prc@wharton.upenn.edu

http://www.pensionresearchcouncil.org/boettner 


\title{
Functional Disabilities and Nursing Home Admittance
}

\begin{abstract}
$\underline{\text { Abstract }}$
This paper examines how inability to perform activities of daily living relates to the risk of nursing home admission over older adults' life courses. Using longitudinal data on persons over age 50 from the Health and Retirement Study, we show that aging one year boosts the probability of having two or more disabilities by 9 to 12 percent in a multivariate logistic model. Moreover, at least three-fifths of all 65-year-old men and three-quarters of women will experience disability levels during their remaining lifetimes severe enough to trigger nursing home admission. Our analysis also suggests that certain types of disability are more important than others in predicting nursing home admittance and use, which has implications for the design and benefit triggers for long-term care insurance programs.
\end{abstract}

Joelle H. Fong (corresponding author)

ARC Centre of Excellence in Population Ageing Research

University of New South Wales, Sydney, Australia

h.fong@unsw.edu.au

Benedict SK. Koh

LKC School of Business

Singapore Management University

skkoh@smu.edu.sg

Olivia S. Mitchell

NBER and The Wharton School, University of Pennsylvania

Philadelphia, PA, USA

mitchelo@wharton.upenn.edu 


\title{
Functional Disabilities and Nursing Home Admittance
}

\author{
Joelle H. Fong, Benedict SK. Koh, and Olivia S. Mitchell
}

Health status is one of the most important determinants of demand for nursing home care in the older population. When physical or mental health deteriorates, or because of memory problems, an older individual may become less able to perform basic human functions such as bathing, dressing, or eating, that people in normal health take for granted. Standard measures of these basic functions or so-called "activities of daily living" (ADLs) are widely used to evaluate health status in relation to nursing home care needs. Public and private long-term care (LTC) insurance programs use information on ADLs to determine individuals' eligibility for care.

Numerous studies show that older adults who need assistance with at least one basic ADL disability are more likely to need nursing home care, compared to those without; moreover, this risk is positively related to the number of ADL disabilities at baseline (Liu, McBride, and Coughlin, 1994; Wallace et al., 1998; Akamigbo and Wolinsky, 2006; Gaugler et al., 2007). As such, it is not surprising that many private long-term care insurance policies in the U.S. as well as the public Medicaid program determine reimbursement eligibility for long-term care expenditure based on a count of ADL disabilities. ${ }^{1}$ In general, persons who require substantial assistance in performing at least two of six ADLs for at least 90 days qualify for insurance payouts (Wiener et al., 2000; Stone, 2002). The ADLs referenced typically include bathing, dressing, eating, toileting, transferring to/from a bed or chair, and walking. These health-related "benefit triggers" can differ across insurers and countries: for instance, the national LTC

\footnotetext{
${ }^{1}$ Since the U.S. Medicaid is a health insurance program for the indigent, Medicaid enrollees must also meet stringent asset and income eligibility criteria in order to be eligible for long-term care services paid for by Medicaid. Other than counting ADL disabilities, private insurers may also pay out benefits in cases where the individual needs substantial supervision due to severe cognitive impairment.
} 
insurance program (called "ElderShield") in Singapore pays out cash benefits only if a person has difficulty in performing at least three ADLs. ${ }^{2}$

This paper examines the interaction between ADL disabilities among U.S. older adults and the risk of nursing home admittance. First, we evaluate the proportion of older individuals that meets the two-plus, or a stricter three-plus, ADL benefit trigger commonly set by insurers. Trends in functional disability over the life course provide insights as to when the elderly are most likely to suffer from ADL disability levels that potentially trigger nursing home use (and may qualify them for LTC insurance benefits). Further, our emphasis on life-cycle ADL prevalence rates complements prior studies examining how functional disability has changed over time and across cohorts in the U.S. We also assess prevalence rates for six specific ADL types. Second, we quantify the impact of ADL disabilities on the risk of eventual nursing home admission using a duration model that takes into account the competing risk of death. Of interest is not only how the risk of institutionalization varies with the number of ADL disabilities, but also with each of the specific ADL types. In the same vein, we explore whether some ADLs are better predictors of length of time in a nursing home until death, compared to others.

A key contribution of this paper is that we determine the relative importance of six basic activities of daily living in predicting nursing home admittance and use. In many countries, the "benefit triggers" used in LTC insurance programs implicitly assume that the six ADLs deserve equal weighting or importance in driving the demand for nursing home care, but this is not necessarily accurate. Some medical studies, for instance, suggest that there may be a hierarchical structure to the order in which loss of ability in ADL items occurs. For example, Katz et al. (1963) report that older persons lose the ability to bathe independently first, followed by dressing,

\footnotetext{
${ }^{2}$ The six ADLs defined by ElderShield are washing, dressing, feeding, toileting, mobility and transferring. The ElderShield is a nation-wide insurance program covering most Singaporeans and permanent residents. LTC policies under this program are sold by commercial insurers who are pre-determined by the government.
} 
toileting, transferring, continence, and eating, in that order. Later research finds broadly similar results: bathing and walking are among the first activities with which older adults have difficulty and eating is the last (e.g. Dunlop et al., 1997; Jagger et al., 2001; Kingston et al., 2012). ${ }^{3}$ If so, then we might anticipate that certain ADLs will be better predictors than others of nursing home admittance outcomes. Accordingly, it is valuable to distinguish the relative importance of each ADL disability and evaluate how this ordering relates to the pattern by which older people lose functional capacity.

A large body of research focuses on how age-adjusted chronic disability among older Americans has changed over time and across cohorts. Most analysts agree that, for adults age $65+$, there have been improvements in many measures of late-life functioning (see e.g., Cutler, 2001; Crimmins, 2004; Freedman et al., 2004; Manton, Gu, \& Lamb, 2006). Health and functioning measures analyzed include chronic health conditions (e.g. diabetes, cancer), impairments (represented by physical functional limitations like climbing stairs, stooping, etc.), ADLs, and instrumental activities of daily living (IADLs) such as shopping for groceries. This decline in late-life chronic disability among older Americans has important implications for the long-term care insurance market. In particular, it could be part of the reason why the U.S. private LTC insurance market is so small. ${ }^{4}$ Our analysis aims to contribute to this debate by reviewing ADL disabilities among the elderly vis-à-vis the reimbursement eligibility or level of care criterion currently used by insurers. In particular, there could be age-specific or sex-specific

\footnotetext{
${ }^{3}$ The inventory of ADLs varies across surveys and over time. Notably, 'walking' is typically listed as an ADL item instead of 'continence' in most surveys such as the National Health and Nutrition Examination Survey (NHANES). Continence may still be evaluated within the survey but not as an ADL item.

${ }^{4}$ For example, Brown and Finkelstein (2007) report that only 10\% of the elderly in U.S. has some form of private LTC insurance. In addition, Brown and Finkelstein (2009) suggest some demand- and supply-side factors rationalizing the small size of the private LTC insurance market, such as high premiums and loads, limited consumer knowledge, and potential substitutes for private formal insurance.
} 
differences in the specific ADL types, which may help LTC insurers define potential markets of policy purchasers and thus promote growth in the private LTC insurance markets.

In what follows, we first review current criteria for admittance to nursing home facilities in the U.S., as well as reimbursement eligibility under private and public LTC insurance programs. We then examine the prevalence of ADL disabilities using a longitudinal panel data set of older adults in the U.S. Health and Retirement Study, and we quantify the probability of older individuals ever developing two-plus ADL disabilities over their lifetimes.

\section{Nursing Homes and Eligibility for Long-term Care}

Nursing home facilities cater to individuals who are ill, frail, and elderly, and who require nursing care or protective supervision. Such residential facilities - offered by public institutions or private providers - provide various levels of care that can range from custodial to skilled nursing delivered by trained professionals to assist persons with physical, emotional or mental impairments. These also encompass long-term hospitals, nursing facilities, intermediate care facilities, and other residential care facilities. In the U.S. and many other countries, nursing homes must be licensed and certified by state and federal government agencies. Long-term institutionalization is typically defined as stays in a nursing facility for 30 consecutive days or more (Friedman et al., 2005). By contrast, short-term stays such as those for persons seeking temporary respite and hospice care are not considered to be institutionalization, inasmuch as they do not amount to extended durations.

Since institutional care is expensive, individuals who require long-term stays in nursing homes often also rely on financial reimbursements from public or private sources to help cover the care costs of nursing facilities. Medicaid, a U.S. federal-state health insurance program for 
the indigent, is the primary source of public funds for long-term care expenditures and reimburses approximately $35 \%$ of LTC expenditures for all elderly (CBO, 2004). ${ }^{5}$ A considerable US\$106 billion was spent by Medicaid on LTC services for elderly and disabled individuals in 2008 (Deloitte, 2010); moreover, the rate of growth of such expenditures is projected to rise at 7.5\% annually (Truffer et al., 2010).

Because Medicaid is a means-tested program intended to provide assistance to persons who are unable to afford necessary medical and health-related services, there are stringent asset and income eligibility criteria. Aged and/or disabled individuals are also assessed in terms of whether they are eligible for Medicare-paid long-term care services. The specific measures used to determine an applicant's eligibility for long-term care services may vary across states, but the fundamental considerations include an assessment of the applicant's medical and nursing needs, mental and physical impairments, and ability to carry out ADLs and IADLs (Stone, 2002). The typical list of ADLs includes bathing, dressing, eating, getting around inside the home (or walking across a room), toileting, and transferring (from a bed to a chair). IADLs refer to tasks necessary for independent community living such as shopping, light housework, telephoning, money management, and meal preparation. Most other developed countries have similar public health assessments for residential care. In Australia, for instance, individuals are assessed and approved for residential care by an Aged Care Assessment Team (made up of local doctors, nurses, and social workers) before they may be admitted to Government-subsidized care facilities. A similar approach is taken in Japan (Mitchell et al., 2008).

Aside from public funding sources, an institutionalized individual with a private LTC insurance policy may also seek reimbursement when needing care. In general, LTC insurance

\footnotetext{
${ }^{5}$ In contrast, Medicare - the U.S. public health insurance program for the elderly - covers health care for people age 65 and above or who are disabled. However, it is primarily designed to help beneficiaries recover from acute illnesses rather than to provide for long-term care per se.
} 
benefits in the U.S. are only "triggered" when a person needs substantial assistance in performing at least two of six ADLs and the assistance is expected to last at least 90 days, or requires substantial supervision resulting from a severe cognitive impairment (Wiener et al., 2000). These health-related "benefit triggers" are put in place by insurers partly to differentiate between long-term versus short-term care, and partly to help control their costs.

In sum, while there is no unique criterion used in determining who is eligible for nursing home admittance, most institutionalized individuals are those who meet the "benefit triggers" of having two-plus or three-plus ADL disabilities. An assessment of ADL disabilities thus helps anticipate the level of care needed for individuals across their life course, and it also provides a widely-used and reasonable gauge of the risk of nursing home admittance.

\section{Measuring the Prevalence of ADL Disability among Older Adults}

To evaluate the extent of ADLs in the older population, and the links to nursing home care, we use the most recent data available from the U.S. Health and Retirement Study (HRS). This is a panel study of older adults (age 50+) who have been interviewed every two years from baseline. It offers a rich source of information on respondents' economic status, physical and mental health, retirement, cognition, pensions, and family structure. Older waves of HRS data were used in prior research to analyze health and functioning including ADL disabilities among older adults (Soldo et al., 2007, Weir, 2007, Freedman et al., 2011). ${ }^{6}$

The HRS periodically adds new birth cohorts of survey participants (and their spouses) to "refresh" the sample of people over the age of 50s. For the present study we use five birth cohorts interviewed in 2010, as follows:

\footnotetext{
${ }^{6}$ All variables are obtained from the RAND HRS data set, version L (RAND, 2011).
} 
- The Asset and Health Dynamics of the Oldest Old (AHEAD) birth cohort was born in 1923 or earlier. Respondents were age 70+ when first interviewed in 1993, and they were at least age 87 if alive in 2010.

- The Children of the Depression (CODA) birth cohort was born 1924-1930. Respondents were age 68-74 when first interviewed in 1998, and age 80-86 if alive in 2010.

- The HRS birth cohort was born 1931-1941. Respondents were approximately age 51-61 when first interviewed in 1992, and age 69-79 if alive in 2010.

- The War Baby (WB) birth cohort was born 1942-1947. Respondents were age 51-56 when first interviewed in 1998, and age 63-68 if alive in 2010.

- The Early Baby Boomers (EBB) birth cohort was born 1948-1953. Respondents were age 5156 when first interviewed in 2004, and age 57-62 if alive in 2010.

In all cases, respondents had to be living in the community when they were first interviewed at baseline; thereafter they were followed into nursing homes and other residential settings. Dates of death were obtained from governmental Vital Statistics sources, when relevant.

The HRS asks about several types of activity limitations in the baseline interview as well as thereafter, covering physical functional impairments (with questions on strength, mobility, and fine motor skills), as well as ADL and IADL disabilities. The six basic ADLs assessed in the survey include dressing, walking, bathing, eating, transferring in/out of bed, and toileting. We define ADL disability in the present paper as having difficulty with any of these activities. ${ }^{7}$ Specifically, respondents are asked whether they have difficulty with ADLs in the following manner:

Here are a few more everyday activities. Please tell me if you have any difficulty with these because of a physical, mental, emotional or memory problem. Again exclude any

\footnotetext{
${ }^{7}$ Some studies distinguish between definitions or measures of disability by asking whether people "have difficulty", "get help", and "get help or use equipment" (see Freedman et al., 2004).
} 
difficulties you expect to last less than three months. Because of a health or memory problem do you have any difficulty with [ADL]?, where [ADL] refers to six different activities, namely dressing, including putting on socks and shoes; walking across a room; bathing or showering; eating, such as cutting up your food; getting in and out of bed; and using the toilet, including getting up and down.

If the person responds 'yes', 'can't do', and 'don't do' to each of these six questions, we coded him as having a disability. ${ }^{8}$

It is worth noting that the specific wording of the ADL question, number of activities assessed, and response collection, has been consistent only from wave 1998 onwards. Consequently, we exclude waves 1992 and 1994 (for the HRS birth cohort) since the question wording was quite different and the toileting ADL was not evaluated. We include waves 1993, 1995, and 1996 in our analysis since the ADL question wording is almost identical to that of wave $1998 .^{9}$

Since 1995, HRS has collected information about whether the respondent resided in a nursing home or other health care facility at the time of the interview. Other health facilities include those which provide all of the following services for its residents: dispensing of medication, 24-hour nursing assistance and supervision, personal assistance, meals, and room. This residence status is noted by the interviewers, but where necessary, respondents are asked:

\footnotetext{
${ }^{8}$ See HRS (2004) and Freedman et al. (2011). Wave-specific responses from waves 1993/1994 through 2010 are obtained from the RAND HRS data. RAND recoded ADL responses in earlier waves to ensure consistency across waves. Responses such as 'refuse to answer' or 'don't know' are classified as invalid responses (on average only $0.3 \%$ per wave).

${ }^{9}$ The HRS implemented a new set of skip patterns in the ADL question sequence from wave 1998 on, whereby respondents could "skip" the ADL questions if responses to preceding (screening) questions on strength, mobility, and fine motor skills suggest that they are high-functioning individuals (HRS, 2004). Respondents could also "skip" the ADL questions if they reported at most one problem in the screening questions and had no difficulty with the dressing ADL. In contrast, ADL questions were universally asked in waves 1993 and 1996. A subset of the skip patterns was implemented in wave 1995. To examine whether the ADL responses in these three waves are comparable to those of later waves, we conducted a separate robustness analysis by retrospectively applying the wave 1998 ADL screening technique to the earlier waves, and assessing whether the ADL responses change significantly. We find no substantive differences between the original and retrospectively-screened ADL responses for these waves, mainly because the bulk of those who had no difficulties with the strength, mobility, and fine motor skills tasks also reported no difficulties with all of the ADLs. This is consistent with HRS' documentation for the 1995 interview, that almost all respondents who could perform the physical functioning tasks in that wave could also perform the ADLs (HRS, 2004). Accordingly, we rely on the original ADL responses in the three identified waves since they are broadly comparable with later waves' responses.
} 
"Are you living in a nursing home or other health care facility?" We use the first instance of a positive response as an indicator of nursing home admission (i.e. long-term, continuous nursing home stays), provided stays in the nursing home lasted for 30 consecutive days or more. To evaluate length of stay, we rely on a combination of interview dates, nursing home residence status in adjacent waves, and where available, self-reports on the month and year that the respondent moved to the nursing home. Responses to separate questions on short-term, overnight nursing home stays are not used.

\section{Analytic Approach}

To examine how the prevalence of ADL disabilities varies over the lifecourse for older individuals, we graph three outcomes by years of age: any ADL, two-plus ADLs, and three-plus ADLs. All five birth cohorts are used, first on a cross-sectional basis, and then on a longitudinal basis. For the cross-sectional analysis, we select the latest interview wave (i.e. wave 2010) and present age-specific prevalence rates for five-year age groups $(55-59,60-64,65-69$, and so on to 100+). Only data points containing valid responses for all six ADL tasks are used, since it is necessary to tally the number of ADL disabilities for each observation. Non-respondents in each wave, and cases with incomplete ADL information, are excluded from both the numerator and denominator when calculating prevalence rates. ${ }^{10}$ Given that the community-based population is likely to be less severely disabled than the institutionalized population, we present additional graphs for the community-based population only. The wave 2010 cross-sectional sample comprises a total of 14,404 subjects, of which 13,950 are community-based.

\footnotetext{
${ }^{10}$ Our procedure departs from Freedman et al. (2004) where cases with missing ADL information were included in the denominator but excluded from the numerator. The maximum possible sample per wave is made up of survey participants who are alive and non-attrited. In each wave, there is a small proportion which does not respond (ranging from 1.1 to $13.6 \%$ per wave); among those who do respond, a handful of participants provide invalid responses (ranging from 17 to 139 per wave, and on average only $0.3 \%$ per wave).
} 
For the longitudinal analysis, we use HRS waves 1993 through 2010. Respondents with complete data on all six ADLs in any one wave are pooled and then sorted into the five-year age groups. The sample of older adults observed over the 10 waves comprises 27,994 individuals (ranging from 6,395 to 20,412 per wave). ${ }^{11}$ Graphs are presented for the total sample as well as for the community-based subsample. To test for age and sex differences in ADL disability prevalence, we also fit binary logistic regression models that control for age, age-squared, sex, interactions of the age variables with sex, and birth cohort dummies. Regressions are performed separately for each outcome (any, two-plus, and three-plus ADL disabilities). The cross-sectional and longitudinal samples are unweighted to accommodate institutionalized individuals (who are assigned zero weights post-institutionalization in the survey waves).

\section{Results: Prevalence of ADL Disability}

Cross-sectional results appear in Figure 1, which shows the prevalence of ADL disabilities (any, two-plus, and three-plus) for the cross-sectional sample of HRS survey respondents of different ages. Fewer than 10 females (and no males) are observed above age 100 . Panel A shows that less than one-third of individuals has any difficulty with ADL functions below age 80 , regardless of sex. This percentage increases sharply after age 80 . Conditional on surviving to age 90, almost three-fifths of all females (and half of all males) have at least one ADL disability. A smaller proportion of males suffer from ADL disabilities than females at advanced ages, possibly because those males who manage to survive to very old ages tend to be the hardier ones. In contrast, females have longer life expectancies, so those who reach age 90 are not necessarily healthier.

[Figure 1 here]

\footnotetext{
${ }^{11}$ The range is quite wide because birth cohorts were added over time. Specifically, fewer subjects were observed in waves 1993 and 1995 (where only the AHEAD birth cohort was interviewed) and in wave 1996 (where only the HRS birth cohort was interviewed), as compared to later waves.
} 
Panels B and C of Figure 1 are of particular interest since these represent the proportion of the sample that would likely require nursing home care and for LTC coverage, depending on the "benefit trigger" criterion used by insurers. Under a two-plus criterion, about 15 to 19 percent of individuals aged 80-85 are estimated to need and qualify for subsidized nursing home care. In comparison, only approximately 9 to 12 percent of individuals would qualify based on the stricter three-plus criterion. The prevalence levels of two-plus ADL disabilities changes most dramatically beyond age 90 for females (and age 85 for males), which suggests that substantial proportions of individuals qualify for subsidized nursing home care quite late in their lives. For females who survive to ages 95-99, approximately two-thirds (67\%) will be eligible for LTC based on a two-plus ADL criterion; one-third of same-aged males will be eligible. Imposing a stricter three-plus criterion for the same age group does not change the proportion of eligible males substantively, but it does reduce the proportion of eligible females to around half (52\%).

If the institutional individuals are removed from the cross-sectional sample, prevalence at each age group decreases by roughly 0.03 percentage points (for each outcome). Nonetheless, the overall trends in ADL disability prevalence over the life course by sex are broadly similar to those of the total sample. Prevalence of functional disabilities among a purely community-based population has been investigated in earlier studies. For instance, using the HRS 2008 wave, Freedman et al. (2011) report that the prevalence of any ADL disabilities was 14.4\% (among the age $65-74$ ), $22.9 \%$ (age 75-84), and 41.9\% (age 85+). Here, using a slightly older sample, our estimated age-specific prevalence rates for narrower age groups are 15.5\% (averaging the 65-69 and 70-74 age groups), $24.0 \%$ (average for those $75-79$ and $80-84$ ), and $48.2 \%$ (for ages $85+$ ), and thus broadly consistent. 
Turning to the longitudinal analysis, Figure 2 portrays the pattern of ADL prevalence for the HRS survey respondents pooled over all waves and age groups; these graphs are much smoother due to the larger sample size. Moreover Figure 2 starts at with the 50-54 age group, and we have a larger sample size for advanced ages (e.g. more than a hundred observations for the $100+$ age group) compared to Figure $1 .{ }^{12}$ Regardless of the outcome measured (any, two-plus, or three-plus ADL disabilities), smaller proportions of males suffer from ADL disabilities than females for most ages. We also conclude that an average older male can likely spend his retirement years without much worry of being inflicted with ADL disability levels that trigger nursing home use. At ages 75 and below, prevalence rates of two-plus disabilities are below 10\%. Between ages $75-85$, at most 10 to $15 \%$ of males have two-plus disabilities. The life expectancy at birth for males is only 75.4 years of age, while that for a male surviving to age 75 is 85.6 (NCHS, 2011).

\section{[Figure 2 here]}

The longitudinal picture is less rosy for females. At ages below 75, the prevalence of two-plus disabilities among females is actually low and very similar to that of males. Prevalence levels, however, start to diverge between the sexes past age 75. Specifically, the overall rate of increase in prevalence level of two-plus disabilities between ages 75 and 85 is larger for females than for males. By age 85 , almost $20 \%$ of females have two or more disabilities. Women are also more likely to live past 85 to advanced ages; the life expectancy for a female conditional on surviving to age 75 is age 87.5 (NCHS, 2011). Consequently, about $30 \%$ of females in the $85-89$ age group are estimated to need and qualify for subsidized nursing home care (compared to $22 \%$ of males). The steepest increase in prevalence levels for women occurs between ages 80-89. This

\footnotetext{
${ }^{12}$ Observations in the age group 50-54 are from the War Baby and Earlier Baby Boomers birth cohorts. Our sample excludes the 1992 and 1994 waves of the HRS birth cohort; see text.
} 
has implications for nursing home use and funding for long-term care. Beyond age 75, women tend to require more nursing home resources and funding than men because they live longer and also because the debilitating condition of two or more ADL disabilities spreads more rapidly among older females than older males for the 75-95 age range.

Next we use binary logistic regressions to examine the relationship between ADL disability and age, sex, and cohort. In Equation (1), Any $D I F F_{i t}$ refers to the probability that the $i$ th survey respondent has difficulty with at least one out of six ADL tasks as of time $t$ :

$$
\begin{aligned}
\text { Any DIFF }_{i t}= & \beta_{1} \text { Age }_{i t}+\beta_{2}\left(\text { Age }_{i t}\right)^{2}+\beta_{3} \text { Female }_{i}+\beta_{4}(\text { Age } * \text { Female })+ \\
& +\beta_{5}(\text { Age } 2 \text { Female })+\beta_{6-9}(\text { Cohort dummies })+v_{i t}+\varepsilon_{i t},
\end{aligned}
$$

where the dependent variable takes a value of 1 if the respondent has one or more ADL disabilities, and 0 otherwise. In our longitudinal sample comprising both community-based and institutionalized individuals, the mean (unweighted) value of $A n y D I F F_{i t}$ is $19.5 \%$. The five birth cohort dummies are namely, AHEAD, CODA, HRS (ref.), WB, and EBB, as described previously. The cohort dummy takes on a value of 1 if the respondent belongs to a particular birth cohort, 0 otherwise. Separate regressions are also performed for two other outcome variables, namely 'having two-plus ADL disabilities' (mean value of observed outcomes is $11 \%$ ), and 'having three-plus ADL disabilities' (mean value of observed outcomes is 7\%).

Results of the logistic regressions for each of the three outcomes appear in Table 1. First, we see that age and age-squared have strong and statistically significant effects on all three outcomes $(p<.01)$. The positive coefficient on age-squared confirms a significant curvilinear effect of age, as noted in our preliminary plots and also in previous studies on disability (e.g. 
Warner and Brown, 2011). ${ }^{13}$ Second, the sex and the sex-age interaction terms have significant impacts on having 'any disabilities' and 'two-plus disabilities' but surprisingly, not on 'threeplus disabilities'. This indicates that while elderly women are more likely than men to experience lower levels of ADL disabilities, they are about equally prone to severe levels of ADL disabilities. Also, the effect of being older is substantively different for females for any and twoplus disabilities outcomes since the interactions are significant in addition to the sum of the individual effects. Interestingly, respondents from both the AHEAD and CODA birth cohorts are less likely to suffer from ADL disabilities than the reference HRS cohort. This may be due to the fact that the former was likely to be a select group of elderly adults with above-average health; they were around age 70 or older at their first interview, and were not institutionalized initially. Of less importance is the significant coefficient for the EBB cohort dummy in the last column; this may be due to small sample sizes (only a tiny percentage of this youngest cohort is observed with three or more ADL disabilities).

\section{[Table 1 here]}

Overall, the results in Table 1 highlight that age has the strongest relationship with ADL disabilities regardless of severity. Figure 3 (Panel A) illustrates the predicted probabilities by sex for the two-plus disabilities outcome. The solid line shows the risk of having two or more ADL disabilities at different points of the life course for males, and the dashed line reflects the risk for females. Not surprisingly, the hazard increases with age, and the impact of aging by one year is larger for women than men. Specifically, the probability of having two-plus ADL disabilities is estimated to increase on average by $12.3 \%$ per year for females, and $9.0 \%$ per year for males (these percentages are the computed average marginal effects). In conjunction, Panel B of Figure

\footnotetext{
${ }^{13}$ The negative coefficient on age is due to the inclusion of age-squared. Overall, the average marginal effect for "age" (combining both age and age-squared) is positive. In a separate regression without the age-squared term, the coefficient on age is positive.
} 
3 displays the predicted probabilities for three-plus ADLs. Here again, the risk increases with age but at a smaller rate of change than for the two-plus outcome. In addition, the figure shows much narrower differences in probabilities between the sexes: for females, the probability of having three-plus ADL disabilities is estimated to increase on average by $7.9 \%$ per year versus $5.7 \%$ for males.

[Figure 3 here]

Our results have implications for the design of ADL criteria in LTC insurance policies. If an insurer were to adopt a two-plus ADL disabilities criterion, this policy will be significantly more likely to pay for women's LTC care than for men, especially for those policyholders who survive beyond age 80 . On the other hand, a stricter three-plus criterion would make the allocation of resources more equal by sex, since there are no significant sex differentials at more severe ADL disability levels (conditional on survival). Finding no sex differential for the threeplus disabilities outcome, while finding them for lesser disability levels, rejects the notion that elderly women may be systematically more disadvantaged than men because they tend to assess their own health less positively than men (c.f. Hall and Channing, 1990; Arber and Ginn, 1993).

Next we compute the lifetime probability of a 60 -year old ever having at least one ADL disability in his or her remaining years, taking into account relevant mortality information. To this end, we use the National Center for Health Statistics 2007 period life table which features a terminal age of 100 (NCHS, 2011). We conclude that about 866 per 1,000 men who survive to age 60 will develop at least one ADL disability before their terminal age; in other words, the lifetime probability is about $86.6 \%$. The corresponding probability for women is $93 \%$. This concords with an AARP (2003) report that around 68\% of people age 50+ suffer from a longlasting condition (but not necessarily an ADL disability) limiting physical mobility. Conditional 
on living to age 60, the lifetime probability of ever having two-plus and three-plus ADL disabilities is $73.6 \%$ and $61.2 \%$, respectively, for men, and $85.8 \%$ and $76.7 \%$ for women. Estimated conditional probabilities for incidence of disabilities decrease with age due to the competing risk of death, since only those who manage to stay alive continue to be at risk of experiencing disabilities. Thus, of those alive at age 65 , men's estimated lifetime probability of ever having one-plus, two-plus and three-plus ADL disabilities is $84.4 \%, 71.4 \%$, and $59.5 \%$ respectively; for women it is $91.4 \%, 83.7 \%$, and $74.9 \%$ respectively. In other words, at least three-fifths of all 65-year old men and three-quarters of women will experience disability levels during their remaining lifetimes severe enough to trigger nursing home admission.

Figure 4 presents the prevalence for each of the six ADL disabilities by age, for the same longitudinal sample of 27,994 individuals observed for 10 waves. Under age 75 , the pattern is unclear for men, while after age 75 , the three most prevalent disabilities are bathing, dressing, and walking (Panel A). Inability to eat without help is the least prevalent ADL limitation. Transferring and toileting are in the middle, and their prevalence rates are close. For women over age $75+$, the ADLs which are most prevalent are problems with bathing, dressing, and walking. It is of interest that the six lines depicting each ADL disability for women are much more disperse, indicating greater distinction in prevalence rates across ADL types (Panel B). For example, among females age $85-89$, some $29.6 \%$ had difficulty with bathing, $26.6 \%$ had difficulty with walking, and $24.5 \%$ had difficulty with dressing. For men in the same age group, only $20.2 \%$ had difficulty with bathing, $19.2 \%$ had difficulty with walking, and $22.3 \%$ had difficulty with dressing.

[Figure 4 here] 
Our results are fairly comparable to those of earlier studies, allowing for the fact that survey datasets may slightly differ in terms of their inventories of ADLs and the manner of assessment (e.g. differences in question wording or definitions). For respondents below age 65, our results on difficulty with dressing and transferring being most prevalent are somewhat consistent with Martin et al. (2010)'s finding that higher proportions of non-institutionalized individuals in the U.S. National Health Interview Survey between ages 50-64 needed help with bathing, dressing, and transferring (in/out of bed/chair), compared to other ADLs. For respondents above age 65, our finding that difficulty with bathing, dressing, and walking are most prevalent also broadly concords with Crimmins (2004) who also found problems with walking, bathing, and transferring to be most prevalent among Medicare beneficiaries age $65+.{ }^{14}$ In addition, others have shown using different dataset that bathing and walking are the first activities where older adults lose functional capacity (Dunlop et al., 1997; Jagger et al., 2001; Kingston et al., 2012).

\section{A Competing Risk Framework}

To estimate more thoroughly how ADL disabilities influence older persons' risk of entering a nursing home, we use a duration-based hazard model.

\section{Model}

Our goal is to estimate the risk of admission from the community to a nursing home, and those who do enter nursing homes: the risk of death. This model accounts for the competing risk

\footnotetext{
${ }^{14}$ In Figure 4, the dressing ADL curve lies above those of the other five ADLs over a substantial range of ages for both sexes. The higher observed prevalence of dressing ADL in our sample - which is not noted in prior literature could be partly attributable to the HRS screening procedure for the ADL question sequence. Specifically starting from wave 1998, respondents who reported one difficulty in the screening questions (on strength, mobility, and fine motor skills) are asked whether they have difficulty with dressing. Those who answered that they had no difficulty with dressing could "skip" the rest of the ADL questions. Thus in this way, more respondents are assessed on their dressing ADL as compared to other ADLs.
} 
of mortality, that is, the risk that individuals may die before experiencing a transition from the community to the nursing home facility. All initially community-dwelling individuals are assumed to be continually at risk of entering a nursing home until death. HRS respondents who did not enter a nursing home, attrite, or die before the end of the survey period are treated as censored.

The analysis focuses on the subset of survey respondents who were alive, living in the community, and responded to the 1998 HRS survey (which here we term the reference point or baseline). This group includes a mix of respondents from the first four birth cohorts (and it excludes the EBB birth cohort first interviewed in 2004, as described above). We also omit persons for whom vital status information was incomplete, or who gave invalid ADL responses at the baseline interview, or who were long-term nursing home users (previously interviewed in a nursing home or other health care facility) prior to 1998. Accordingly, the analytical sample consisted of 19,952 older adults, with $41 \%$ from the HRS birth cohort, $25 \%$ from AHEAD, 19\% from CODA, and 15\% from WB. Further descriptive statistics for the sample appear in Appendix 1.

Three distinct states are defined in the model: living in the community ('state $C$ '), living in a nursing home at the time of interview ('state $N$ '), and death ('state $D$ '). Figure 5, Panel A illustrates these states and the associated transitions. Consistent with prior literature (e.g. Liu et al., 1994), we assume that the timing of exit from starting state $C$ to state $N$ is statistically independent from the timing of death (i.e. exit to state $D$ ). In other words, deaths are assumed to be occurring randomly and their occurrences are unrelated to the reason for transition from the community into a nursing home or health care facility. Hazard functions can then be estimated separately and the problem of "competing risk" in the estimation is handled as right-censoring 
(Kalbfleisch and Prentice, 1980). We observe that only a very small proportion of the sample (or $0.6 \%)$ exhibit recovery, that is, transit from nursing homes back to the community. As such, for simplicity, we assume that living in a nursing home is persistent after entry.

[Figure 5 here]

Panel B of Figure 5 depicts possible patterns of transition for these 19,952 initially community-dwelling individuals. In the diagram, representative individuals are labeled A to D; the percentage of the sample that each individual represents is shown at the right of the diagram. Person A represents a person who remains in the community and is not reported to be living in a nursing home from 1998 until his last interview; 61\% of the sample falls in this category. Their length-of-stay in the community will be right-censored at the point that they permanently attrite from the study, or by the 2010 interview (the end of the observation period) ${ }^{15}$ Person B depicts a person who died in the community and never transited to a nursing home; around one-third of the sample (32\%) falls in this category, which highlights the importance of modeling the competing risk of mortality in this setup. Their lengths-of-stay in the community are measured as the observed duration from 1998 until death. The remainder of the sample (7\%) moved into a nursing home at some time during the 12-year observation window. Of these 1,437 individuals, 946 were observed to have died while in the nursing home (represented by Person C). These individuals will have both a completed length-of-stay in the community as well as a completed length-of-stay in the nursing home. A last category, Person D, represents subjects who transited to a nursing home but their length-of-stay in the nursing home is right-censored at the point that they permanently attrited or in 2010 . For all persons, the observed duration of stay in the community equals the elapsed time between the 1998 interview and either entry into a nursing home, death, or 2010 cut-off, whichever occurs first.

\footnotetext{
${ }^{15}$ Right-censoring through attrition is not substantial in our sample (less than 3\% over the full 12-year window).
} 
The hazard function is the instantaneous rate of experiencing a given event (e.g. the transition from state $\mathrm{C}$ to N) at time $t$. Following Kalbfleisch and Prentice (1980) and Liu et al. (1994), the hazard function in the presence of competing risks is expressed as:

$$
\begin{array}{r}
h_{j}^{s}\left(t ; Z_{i}\right)=\lim _{\delta \rightarrow 0} \frac{1}{\delta} \operatorname{Pr}\left(t \leq \operatorname{LOS}_{i}^{s}<t+\delta, J=j \mid L O S_{i}^{s} \geq t, Z_{i}\right), \\
\text { for } s=c, n ; j=n, d .
\end{array}
$$

In Equation (2), the function $h_{j}^{s}\left(t ; Z_{i}\right)$ is the specific hazard rate of failure of type $j$ at time $t$ for an individual $i$ starting in state $s$, given personal characteristics $Z_{i}$ and the presence of other failure types. $\operatorname{LOS}_{i}^{S}$ is his length-of-stay in starting state $S$, which could be either state $C$ (community) or state $N$ (nursing home). There are two failure types $(j)$ from state $C$ given by exit to state $N$, or exit to death. Following the assumption that nursing home entry is permanent, death is the only form of exit from state $N$. Accordingly the three hazard functions to be separately estimated, using Cox proportional hazard regressions, are:

$$
\begin{aligned}
& h_{N}^{C}\left(t ; Z_{i}\right)=\text { the hazard rate for exiting from state } C \text { to state } N, \\
& h_{D}^{C}\left(t ; Z_{i}\right)=\text { the hazard rate for death in the community, } \\
& h_{D}^{N}\left(t ; Z_{i}\right)=\text { the hazard rate for death in the nursing home. }
\end{aligned}
$$

We examine two configurations of functional disabilities in the ADL set. First, we count each individual's number of ADL disabilities. This is informative since many LTC policy benefit triggers use a count of disabilities. Also some prior studies examining the risk of nursing home placement have modeled ADL limitations by count, either as a scale variable (e.g. ranging from 0 to 6 ) or as categorical variables (e.g. $<2$ and $\geq 2$ disabilities). To this end, we create a total of seven dummy variables corresponding to 0 disability (ref.), 1 disability, 2 disabilities, and so on.

A second configuration disaggregates and tests separately each of the six disability types. This follows from our conjecture that certain disabilities may be more important in driving the 
demand for nursing home care. Furthermore, not all the six activities may necessitate skilled, formal, trained nursing care provided in nursing homes. For instance, difficulty with walking may be eased considerably through the use of aids like a walker, cane, or rails, but it may be hard to bathe independently even with grab bars. Another aim is to assess whether those activities which individuals first lose functional capacity in and most prevalent at older ages, specifically bathing and walking, are also those which are most predictive of nursing home use.

The analysis also controls on other independent variables previously shown to be risk factors for nursing home admission among U.S. older adults (Liu et al., 1994; Friedman et al., 2005; Akamigbo and Wolinsky, 2006). These include predisposing characteristics (age, sex, and race), enabling factors (marital status, and coverage by long-term care insurance), and need (ever-have chronic conditions, and cognitive status). All regressions are weighted using baseline individual-level weights.

\section{Results: Number of ADL Disabilities}

Table 2 presents the estimates of the effect of number of ADL disabilities on nursing home admission, controlling on other factors. Estimated hazard ratios and $95 \%$ confidence intervals are reported. A hazard ratio larger than unity for the covariate indicates that the covariate is associated with a higher risk of exit, thus shorter duration in the initial state before transition. Results show that females have shorter duration in the community than males and so are more at risk of entering a nursing home facility. Being older is also associated with a higher risk of exit to nursing homes. The number of ADL disabilities measured at the 1998 baseline have independent, significant effects on eventual nursing home admission $(p<.01)$. Not surprisingly, having more baseline ADL disabilities is associated with greater risk of eventual nursing home entry. Specifically, persons with two baseline disabilities are 2.38 times more 
likely to enter a nursing home than those without a disability (able individuals), and persons with six baseline disabilities face 4.29 times the admission risk of able individuals.

[Table 2 here]

A different way to look at the risk of nursing home entry allows for the number of disabilities to vary with time instead of being fixed at baseline. The rightmost columns of Table 2 show that individuals with exactly five disabilities at any point in time are most at risk of entering a nursing home over the 12-years observation span. They are almost eight times more likely to enter a nursing home than abled individuals. Interestingly, they face even higher admission risk than those persons having all six disabilities. Another at-risk group is the set of individuals with exactly three disabilities, who face greater admission risk than those with exactly four disabilities at all time periods.

This finding highlights the nature of competing risks: a more severely disabled group is apt to be at high risk of dying as well as entering nursing homes in a prospective time period. Also, given that we allow for time-varying disabilities, observations of persons with six disabilities probably accrue from the already-institutionalized (i.e. no longer at-risk of admission). ${ }^{16}$ Another takeaway from the results is that specifying a three-plus ADL criterion in long-term care insurance policies is quite stringent. In fact, under this stricter criterion, individuals having three ADL restrictions would be almost as disabled those with four or six disabilities. In other words, a combined count of ADL disabilities is useful in predicting nursing home admittance, but it does not distinguish between the six disability types - an issue to we turn next.

Results: Types of ADL Disabilities

\footnotetext{
${ }^{16}$ For example, of those in the nursing homes as at the 2006 (2010) interview wave, 39\% (35\%) had six ADL disabilities and 16\% (17\%) had five ADL disabilities.
} 
In Table 3, we enter each of the six ADL disabilities as separate variables in the hazard rate equations. Results for the three hazard functions of interest include the hazard of exiting the community to a nursing home (column 1) or to death (column 2); and the hazard of exiting a nursing home to death (column 3). Focusing first on the ADL disabilities and comparing across columns, it is apparent from the table that difficulty with bathing is the single most important ADL disability driving nursing home admission. Specifically, column 1 indicates that individuals with difficulty with bathing face $82 \%$ higher admission risk $(p<.01)$ than those without such difficulty. Furthermore, difficulty with bathing is a strong predictor of mortality among both community-dwellers as well as nursing homes residents. For instance, nursing home residents with difficulty with showering are $31 \%$ more likely to die than those without such difficulty (see column $3 ; p<.05)$. The relative importance of bathing disability can be partly rationalized by it requiring more human assistance than the other basic tasks of everyday life. For example Wiener and Clark (1990) found that higher proportions of adults age 65+ (whether institutionalized or not) required personal help more in bathing than in other activities. ${ }^{17}$ Medical studies also suggests that adults who need assistance with bathing find the activity to be both physically and emotionally demanding due to pain, anxiety resulting from being naked in front of strangers, being afraid of falling, and discomfort from cold or drafty bathing areas or harsh water spray (Rader et al., 2006).

[Table 3 here]

Table 3 also demonstrates that walking and eating disabilities are associated with a 25\% significantly higher risk of nursing home entry among the community residents (see Column 1). Four of the ADL disabilities (dressing, walking, bathing, and eating) are predictive of death in

\footnotetext{
${ }^{17}$ For example, reviewing data across five U.S. surveys, Wiener and Clark (1990) report that between 4.6-6.9\% of the non-institutionalized persons age 65 and older require personal help bathing, 2.9-4.4\% need similar help dressing, 2.6-4.2\% need help transferring, $2.4-3.4 \%$ need help with toileting, and $0.7-2.5 \%$ need help eating.
} 
the community, and associated with relatively large effects. For example, reporting difficulty with walking or eating increases the risk of death by about $50 \%$. In contrast, only bathing and eating are predictive of death in nursing homes and associated with smaller increases in risk (16$31 \%$ ). Transferring and toileting disabilities are not significant in any of the regressions.

One conclusion from these results is that individuals with the walk, bathe, and eat disabilities have higher risks of entering nursing homes, and they are also more likely to die before even being admitted. Another conclusion is that the ADL risk factors most predictive of nursing home admission and time from admission till death - namely, bathing and eating - are not necessarily activities in which individuals first lose functional capacity based on the known hierarchy of disabilities. In particular, eating is typically ranked as the last activity with which older adults have difficulty, yet it is a disability type (other than bathing) that significantly predicts transition from community to nursing homes, and ultimately death.

Consistent with findings from prior research, many of the demographic variables in the regression are significantly associated with nursing home admission. For example, being older, white, non-married, and a lower cognitive score, are associated with higher risk of exit from the community to nursing home. Individuals with a history of chronic conditions such as diabetes, stroke, or major psychiatric illness are also more at-risk of entering nursing homes eventually. Like Liu et al. (1994), we find that sex is not a significant predictor of nursing home admission. Having LTC insurance is also not predictive of nursing home admission. ${ }^{18}$ Overall, the selected covariates explain much of the variation in nursing home admission among the communitydwellers (adjusted $R^{2}=84.8 \%$ ).

\footnotetext{
${ }^{18}$ The absence of significance for these variables is not due to multi-collinearity. For example, the maximum pairwise correlation for 'female' is 0.24 with 'married', and the maximum pair-wise correlation for 'LTC insurance' is just 0.08 with 'cognition'.
} 
Turning attention to death hazards, males have a higher mortality risk regardless of whether they are in the community or institutionalized. Being older, non-married, having chronic conditions (lung disease and cancer), and a lower cognitive score, are all associated with higher mortality hazards and thus shorter duration in the community (column 2). In contrast, a smaller set of factors is predictive of deaths among nursing home residents (column 3). The overall adjusted $R^{2}$ is low (14\%). Accordingly, two observations are worth highlighting. First, while ADL disabilities are extremely predictive of the risk of nursing home admissions, they lack explanatory power with regard to mortality hazards among nursing home residents (as well as duration in a nursing home). Second, deaths within the community and nursing homes appear to be driven by different risk factors. Mortality among the institutionalized, in particular, may be better explained by factors we cannot capture here, including quality of care, access to care, or availability of social support networks.

Results presented thus far assume that the hazard of the risk changes when someone experiences an ADL disability. For instance, the estimated hazard ratio of 1.25 for walking implies that the instant an individual is hit with such a difficulty, his risk of entering a nursing home increases by $25 \%$. Yet in practice, the effect of difficulties in everyday activities might accumulate over time before eventually resulting in failure (in this case, admission to a nursing home). To test whether delay effects of ADL disabilities are significant in predicting each of the three outcomes, we next allow each ADL disability variable to interact with time. Results not detailed here (but available on request) indicate that the time-varying effects of all the six ADL variables are not statistically significant in predicting nursing home deaths. In the hazard regression for deaths in the community, however, difficulty with eating is associated with both immediate and delayed effects ( $p$-value for the eating time-varying coefficient $=.043)$. In the 
hazard regression for exiting the community to a nursing home, the delay effect of the transferring to/from bed ADL disability is statistically significant ( $p$-value for the transferring time-varying coefficient=.009). Thus, while difficulty with walking, bathing, and eating have immediate effects on the risk of nursing home admission, difficulty with transferring is associated with delayed effects on the hazard.

\section{Determinants of Bathing ADL Disability}

Given that bathing is the single most important ADL disability driving nursing home admission and mortality, we next turn to investigate the determinants of bathing difficulty. We rely on the same longitudinal sample used above. Our binary logistic regression model controls for demographic attributes (sex, age, age-squared, race, married), health status (ever-have chronic disease, self-reported health, cognitive status), and lifestyle factors (smoking and drinking). The dependent variable is 1 if the individual $i$ is observed to have a bathing disability at time $t, 0$ otherwise.

Table 4 provides regression estimates. Unsurprisingly, persons who are older, nonmarried, or have a history of chronic conditions (in particular, stroke, psychiatric illness, or arthritis), are more likely to have a bathing disability. The finding that arthritis is a significant risk factor is consistent with Rader et al. (2006) who note that many experiencing pain during bathing have a history of arthritis, osteoporosis, or joint pain. People with difficulty with bathing also are more likely to be nonwhite, lack LTC insurance, smoked before, have lower cognitive scores, and assess their own health as 'poor' or 'fair'.

[Table 4 here] 


\section{Conclusions}

This paper has examined how disabilities in activities of daily living relate to the risk of nursing home admission over the life course of older adults. One conclusion is that at age 75 and below, the prevalence of two-plus ADL disabilities is relatively low and similar between men and women. But beyond age 75 , prevalence levels of disabilities start to diverge between the sexes. Women ultimately need more nursing home resources than men, because they live longer and also because the prevalence of two-plus ADL disabilities rises more rapidly among females than for men beyond age 75. Moreover, the negative impact of aging on ADLs is larger for women than men. Our multivariate estimates suggest that the probability of having two-plus ADL disabilities rises by $12.3 \%$ per year for females, and $9 \%$ per year for males, after age 50 . Men and women look more similar if a stricter three-plus ADL criterion is used, suggesting that LTC costs would be shared more equally between the sexes by the latter metric.

We also show that three-fifths of all surviving 65-year old male retirees and three-fourths of all surviving 65-year old female retirees will experience disability levels which could trigger nursing home use over their remaining lifetimes. Specifically, conditional on living to age 65 , the lifetime probability of ever having two-plus and three-plus ADL disabilities is $71.4 \%$ and $59.5 \%$ respectively for men, and $83.7 \%$ and $74.9 \%$ for women. Moreover, not all reported ADLs are equivalent in terms of predicting the need for long term care. We show that the three most prevalent disabilities are bathing, dressing, and walking, for older adults age $75+$. By contrast, needing help with eating is the least prevalent ADL limitation. In a competing risk hazard framework, having more baseline ADL disabilities is associated with greater risk of eventual nursing home entry. When we allow for time-varying number of disabilities, people with five disabilities at any point in time are most at risk of entering a nursing home (those more severely 
disabled, say with six disabilities, are apt to be at higher risk of dying in any given time period). Additionally, certain disability types - namely, ADLs for bathing and eating - prove to be more important than others in explaining nursing home admittance and duration in nursing homes. Difficulty with bathing is the single most important ADL disability driving nursing home admission and mortality. People having difficulty with bathing face $82 \%$ higher admission risk than those without; this may be because bathing requires more human assistance and is a more physically and emotionally demanding task than other basic activities of daily living. And last, we show that people reporting the walk, bathe, and eat disabilities have higher risks of entering nursing homes, but they are also more likely to die prior to admission. Also, the ADL risk factors most predictive of nursing home admission and time from admission till death - namely, bathing and eating - are not necessarily activities in which individuals first lose functional capacity based on the known hierarchy of disabilities.

In sum, these findings should be of interest to private and public insurers concerned with the design of benefit triggers used in long-term care insurance programs. Clearly ADL disabilities are extremely predictive of the risk of nursing home admissions. On the other hand, they do not have much explanatory power with regards to mortality hazards among nursing home residents. 


\section{References}

AARP (2003). A Report to the Nation on Independent Living and Disability: Executive Summary, Beyond Fifty Series Report, AARP Public Policy Institute, April.

Akamigbo AB., and F.D. Wolinsky (2006). Reported Expectations For Nursing Home Placement Among Older Adults And Their Role As Risk Factors For Nursing Home Admissions. The Gerontologist. 46(4): 464-73.

Brown, J.R., and A. Finkelstein. (2007). Supply or Demand: Why Is the Market for Long-Term Care Insurance So Small? Journal of Public Economics, 91(10): 1967-91.

Brown, J. R., and A. Finkelstein, (2009), The Private Market for Long-Term Care Insurance in The United States: A Review Of The Evidence. The Journal of Risk and Insurance, 76(1): 5-29.

Congressional Budget Office, CBO (2004). Financing Log-Term Care for the Elderly. April.

Crimmins, E.M. (2004). Trends in the Health of the Elderly. Annual Review of Public Health, 25: $79-98$.

Cutler, D.M. (2001). Declining Disability among the Elderly. Health Affairs, 20: 11 - 27.

Deloitte (2010). Medicaid Long-term Care: The Ticking Time Bomb. The Deloitte Center for Health Solutions, Washington, D.C.

Dunlop, D., S.L. Hughes, and L.M. Manheim. (1997). Disability in Activities of Daily Living: Patterns of Change and a Hierarchy of Disability. Am J Public Health. 87(3): 378-383.

Freedman, V., B. Spillman, P. Andreski, et al. (2011). Trends in Late-Life Activity Limitations: An Update from 5 National Surveys. Demography (forthcoming).

Freedman, V.A., E. Crimmins, R.F. Schoeni, et al. (2004). Resolving Inconsistencies in Trends in Old-Age Disability: Report from a Technical Working Group. Demography, 41: 417 441.

Friedman, S.M., D.M. Steinwachs, P.J. Rathouz, et al. (2005). Characteristics Predicting Nursing Home Admission in the Program of All-Inclusive Care for Elderly People. The Gerontologist. 45(2): 157-166.

Gaugler J.E., S. Duval, K.A. Anderson, and R.L. Kane (2007). Predicting Nursing Home Admission in the U.S: A Meta-analysis. BMC Geriatr. 7: 13.

HRS (2004), Documentation of Physical Functioning Measured in the HRS and the AHEAD, HRS Documentation Report, University of Michigan, December. 
Jagger, C., A.J. Arthur, N.A. Spiers, and M. Clarke (2001). Patterns of Onset of Disability in Activities of Daily Living with Age. Journal of the American Geriatrics Society. 49: 404-409.

Kalbfleisch, J.D., and R.L. Prentice. (1980). The Statistical Analysis of Failure Time Data. New York: John Wiley and Sons.

Katz, S., A.B. Ford, R.W. Moskowitz, et al. (1963), Studies of Illness in The Aged: The Index of ADL, A Standardized Measure of Biological and Psychosocial Function. Journal of the American Medical Association, 185(12): 914-919.

Kingston, A., J. Collerton, K. Davies, et al. (2012). Losing the Ability in Activities of Daily Living in the Oldest Old: A Hierarchic Disability Scale from the Newcastle 85+ Study. PLOS ONE. 7(2): 1-7.

Liu, K., T. McBride, and T. Coughlin (1994). Risk Of Entering Nursing Homes for Long Versus Short Stays, Medical Care, 32(4): 315-327.

Manton, K.G., X. Gu, and V.L. Lamb (2006). Change in Chronic Disability from 1982 to 2004 2005 as measured by Long-Term Changes in Function and Health in the U.S. Elderly Population. Proceedings of the National Academy of Sciences of the United States of America, 103: 18374 - 18379.

Martin, L.G., V. Freedman, R.F. Schoeni, and P.M. Andreski (2010). Trends In Disability And Related Chronic Conditions Among People Ages Fifty To Sixty-Four. Health Affairs, 29(4): 725-731.

Mitchell, O.S., J. Piggott, and S. Shimizutani. (2008). An Empirical Analysis of Patterns in the Japanese Long-Term Care Insurance System. Geneva Papers on Risk and Insurance, 33: 694-709.

National Center for Health Statistics, NCHS (2011). United States Life Tables 2007. National Vital Statistics Reports, U.S. Department Of Health And Human Services, September.

RAND Corporation, (2011), RAND HRS Data Documentation: Version L. Rand Center for the Study of Aging, November.

Soldo, B.J., O.S. Mitchell, R. Tfaily, and J.F. McCabe (2007). Cross-cohort Differences in Health on the Verge of Retirement. In B. Madrian, O.S. Mitchell, and B.J. Soldo (Eds.), Redefining Retirement: How will Boomers Fare? New York: Oxford University Press: $138-158$. 
Stone, J.L. (2002). Medicaid: Eligibility for the Aged and Disabled. Congressional Research Service, Report for Congress No. RL31413. July 5.

Truffer, C.J., S. Keehan, S. Smith, et al. (2010). Health Spending Projections Through 2019: The Recession's Impact Continues. Health Affairs, 29(3): 522-529.

Wallace, S.P., L. Levy-Storms, R.S. Kington, and R.M. Andersen (1998). The Persistence Of Race and Ethnicity in the Use of Long-Term Care. Journals of Gerontology, Series B, Psychological Sciences \& Social Sciences. 53(2): S104-112.

Warner, D.F., and T.H. Brown (2011). Understanding how Race/Ethnicity and Gender Define Age-Trajectories of Disability: An Intersectionality Approach. Social Science \& Medicine, 72(8): 1236-1248.

Weir, D. (2007). Are Baby Boomers Living Well Longer? In B. Madrian, O.S. Mitchell, \& B.J. Soldo (Eds.), Redefining Retirement: How will Boomers Fare? New York: Oxford University Press: 95 - 111.

Wiener, J., J. Tilly, and S. Goldenson, (2000). Federal and State Initiatives to Jump Start the Market for Private Long-Term Care Insurance. The Elder Law Journal, 8(1): 57-99. 
Figure 1: Percentage of the Sample Who Reported ADL Disabilities, by Age (HRS Wave 2010).

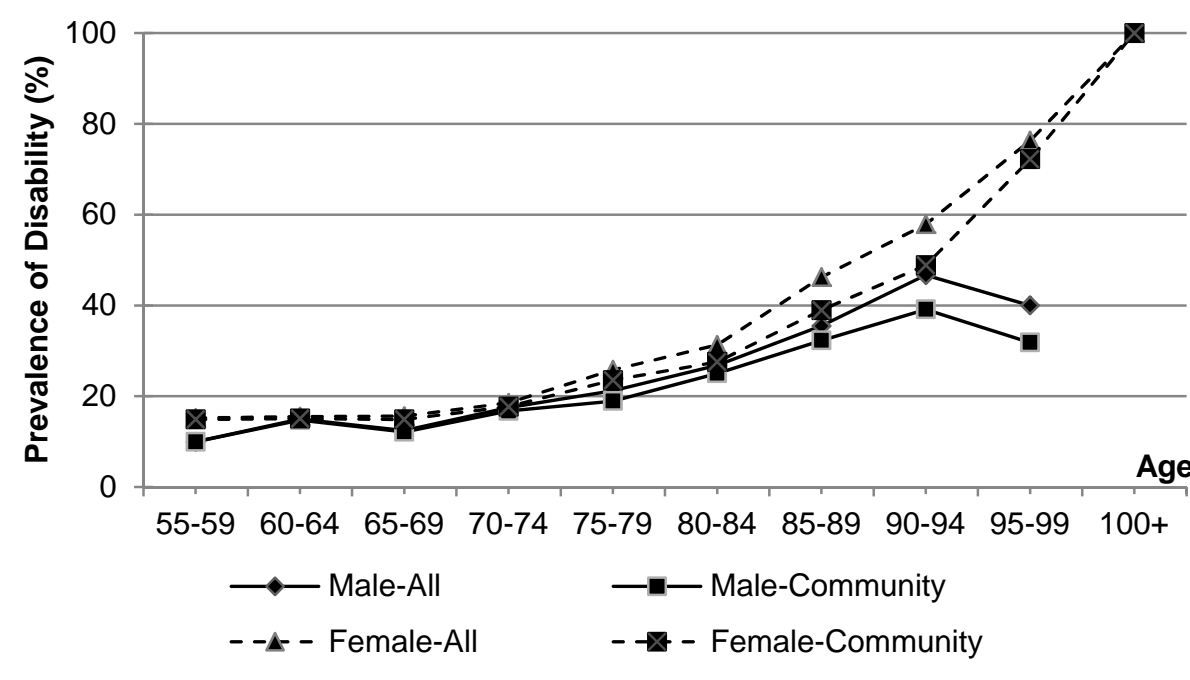

Panel A.

Difficulty with one or more of six ADLs

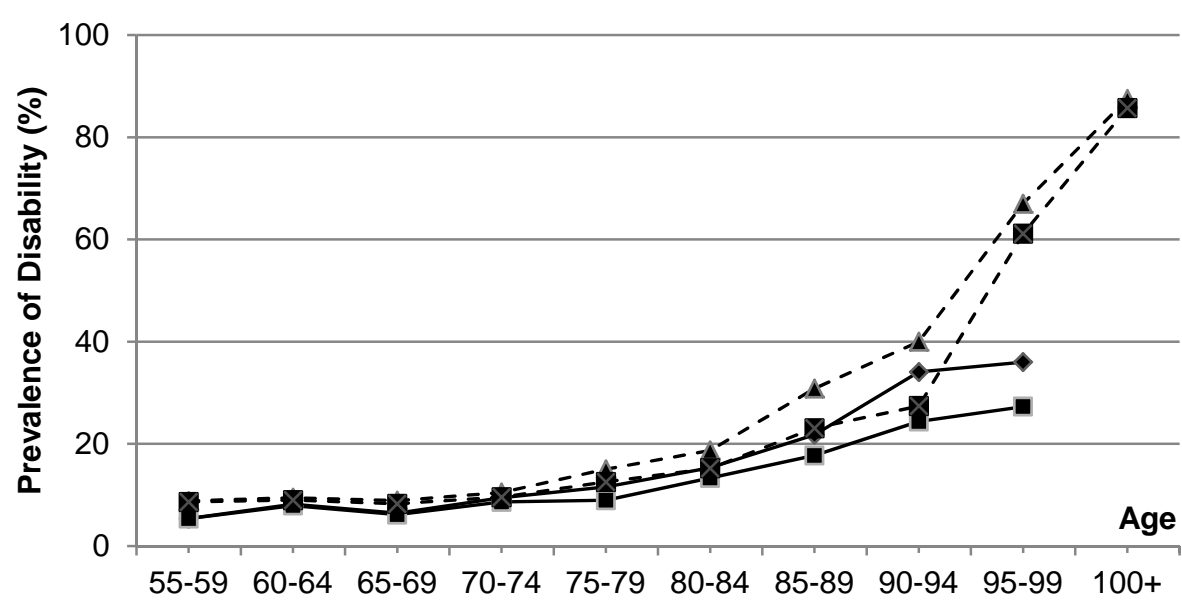

Panel B. Difficulty with two-plus ADLs

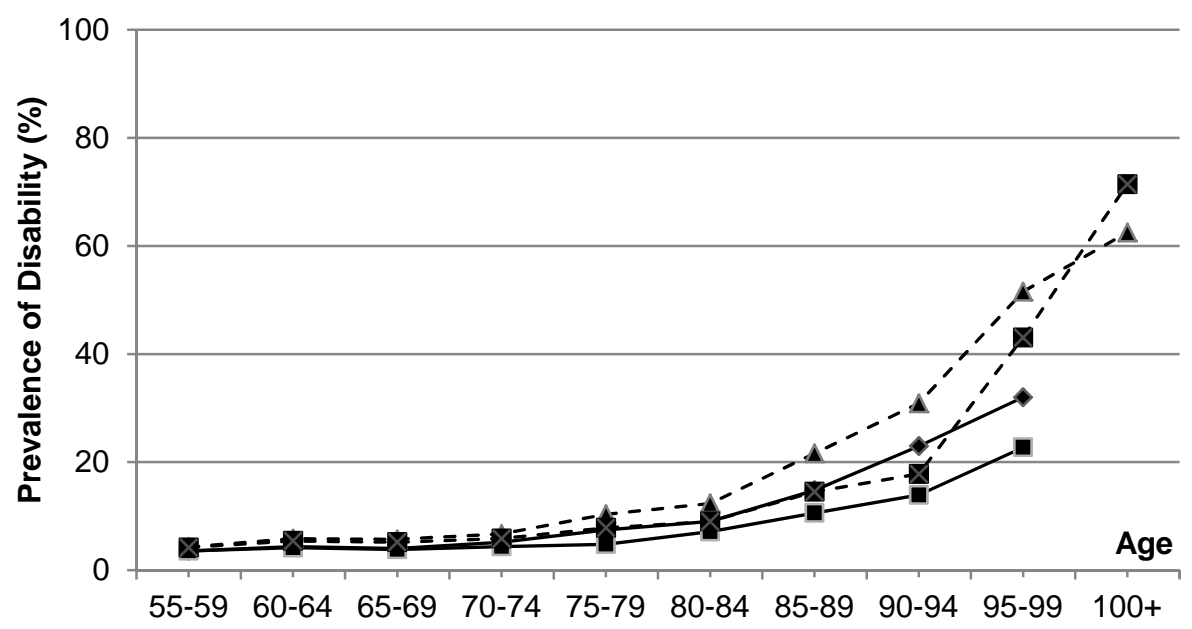

Panel C. Difficulty with three-plus ADLs

Notes: $N=14,404$ subjects, of which 13,950 are community-based. Only data points containing valid responses for all six ADL tasks are used, in order to tally the number of ADL disabilities for each observation. Non-respondents in each wave, and cases with incomplete ADL information, are excluded from both the numerator and denominator when calculating prevalence rate; see text. Source: Authors' calculations. 
Figure 2: Percentage of the Sample Who Reported ADL Disabilities, by Age (HRS Waves 1993 2010).

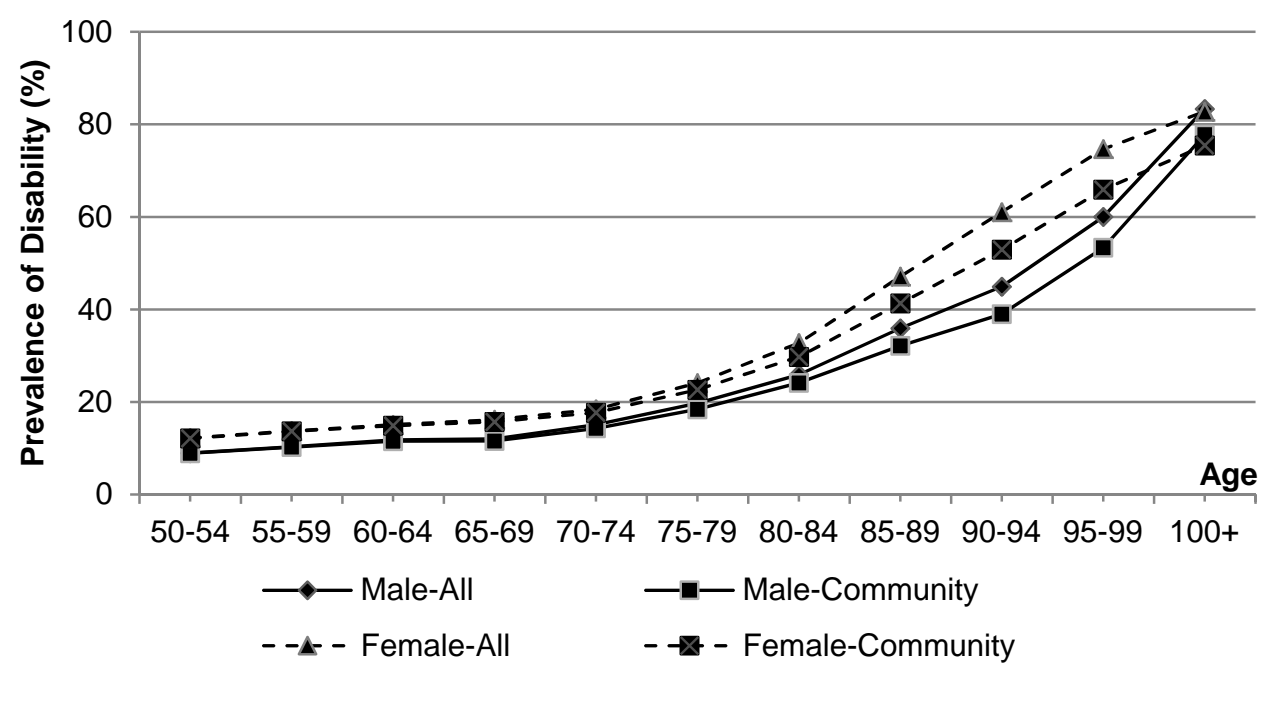

Panel A. Difficulty with one or more of six ADLs

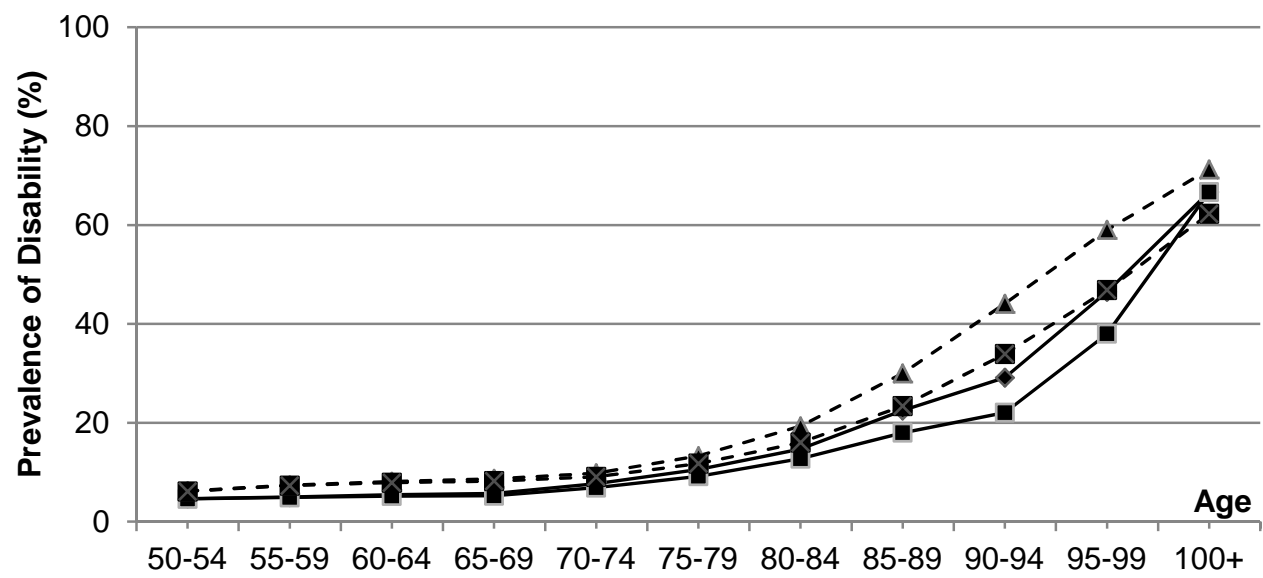

Panel B. Difficulty with twoplus ADLs



Panel C. Difficulty with threeplus ADLs

Notes: $N=27,994$ subjects (ranging from 6,395 to 20,412 per wave). Only data points containing valid responses for all six ADL tasks are used, in order to tally the number of ADL disabilities for each observation. Nonrespondents in each wave, and cases with incomplete ADL information, are excluded from both the numerator and denominator when calculating prevalence rate; see text. Source: Authors' calculations. 
Figure 3: Predicted Probabilities of Having ADL Disabilities, by Age and Sex

\section{A. Two-plus ADLs}



\section{B. Three-plus ADLs}

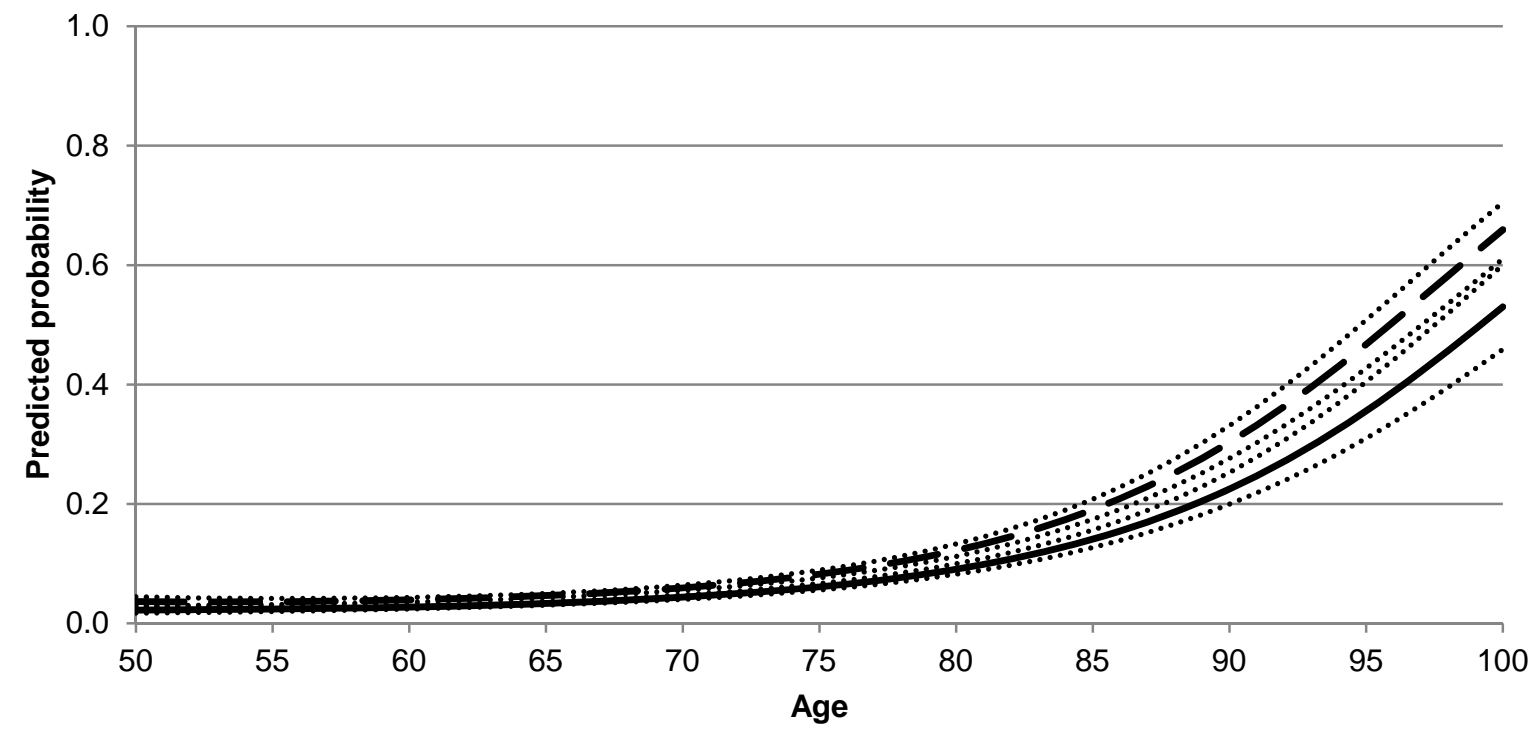

Notes: The dotted lines alongside the curves show the 95\% confidence intervals. Predicted probabilities are obtained from the logistic regression, where the dependent variable takes a value of 1 if the respondent has two or more ADL disabilities, 0 otherwise. Explanatory variables include age, age- squared, female, cohort dummies, and age-sex interaction terms; see text. $N=27,994$ subjects pooled from ten interview waves.

Source: Authors' calculations. 
Figure 4: Percentage of the Sample Who Reported Specific ADL Disabilities, by Age (HRS Waves $1993-2010)$.

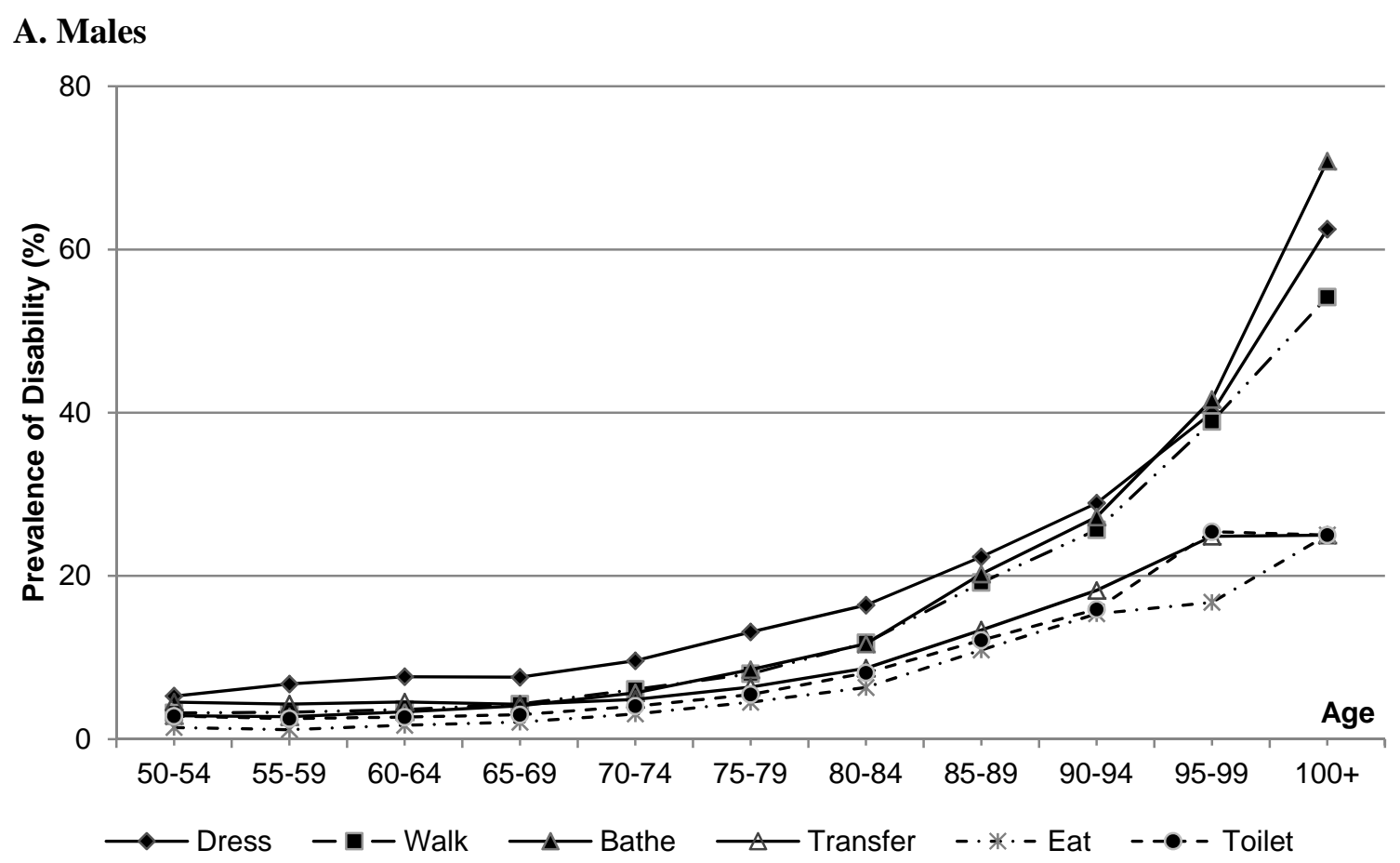

\section{B. Females}

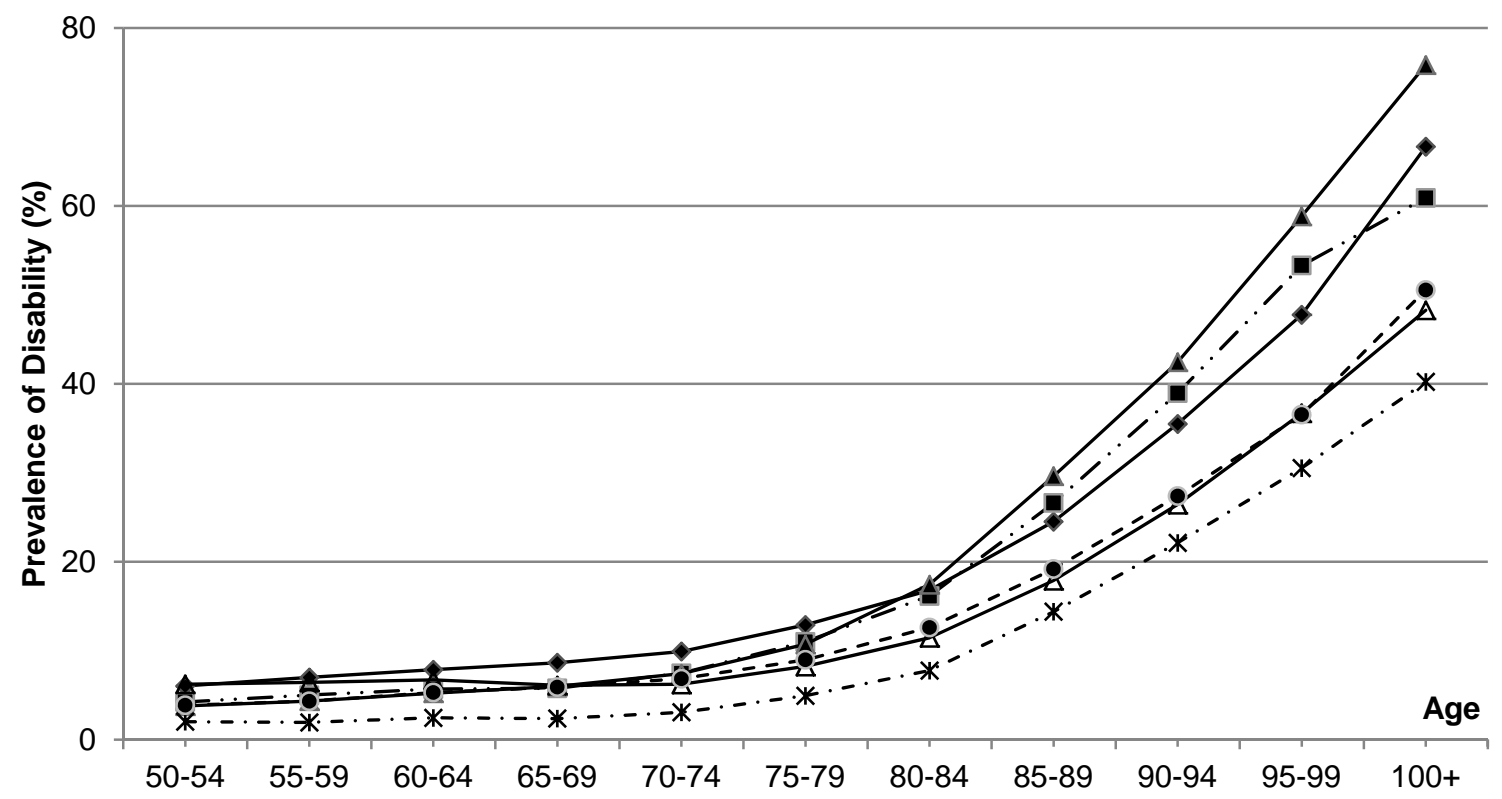

Notes: This Figure shows the proportions of males and females with each type of ADL limitation, by age group. $N=27,994$ subjects pooled from ten interview waves (ranging from 6,395 to 20,412 per wave). Only data points containing valid responses for all six ADL tasks are used, in order to tally the number of ADL disabilities for each observation. Non-respondents in each wave, and cases with incomplete ADL information, are excluded from both the numerator and denominator when calculating prevalence rate; see text. Source: Authors' calculations. 
Figure 5: Competing Risk Model of Nursing Home Admittance

A. Three State Model

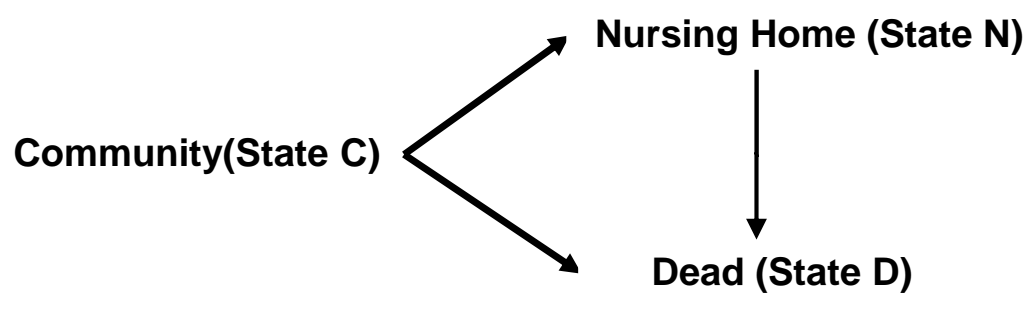

B. Schematic Diagram of Transitions between States

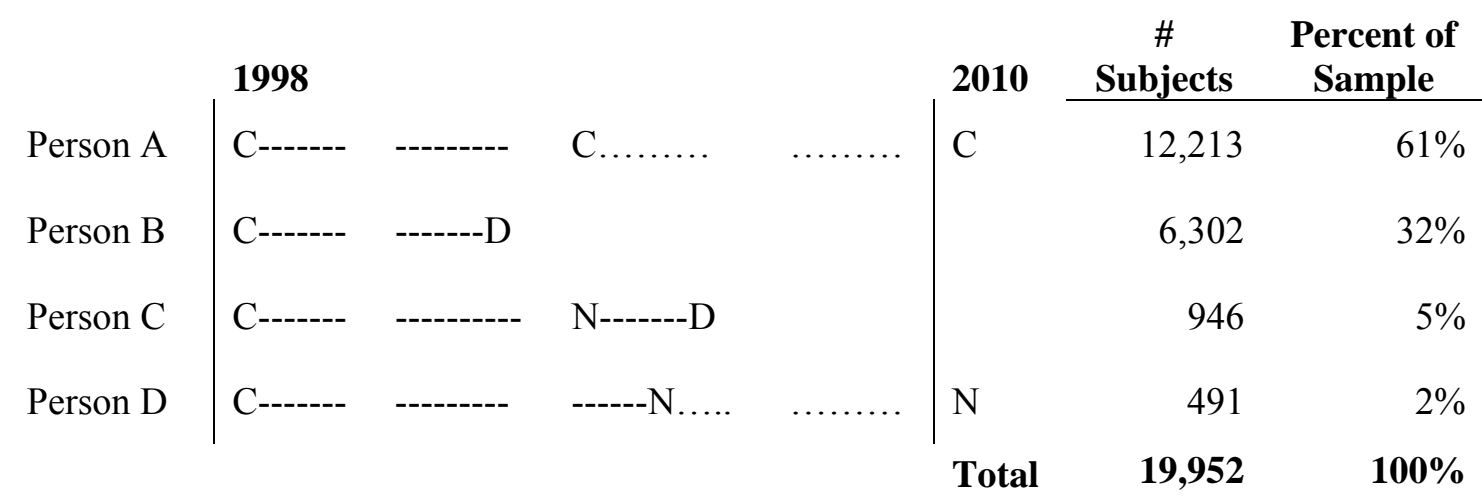

Notes:

State $C=$ Living in community at time of interview

State $N=$ Living in nursing home at time of interview

State $D=$ Dead

Source: Authors'. 
Table 1: Logistic Regression Model Estimation of Probability of Having ADL Disabilities

\begin{tabular}{|c|c|c|c|c|c|c|}
\hline & \multicolumn{6}{|c|}{ Dependent variable: Whether a respondent has... } \\
\hline & \multicolumn{2}{|c|}{ Any ADL Disabilities } & \multicolumn{2}{|c|}{ 2+ ADL Disabilities } & \multicolumn{2}{|c|}{ 3+ ADL Disabilities } \\
\hline & $\begin{array}{l}\text { Estimated } \\
\text { coefficient }\end{array}$ & Std Error & $\begin{array}{l}\text { Estimated } \\
\text { coefficient }\end{array}$ & Std Error & $\begin{array}{l}\text { Estimated } \\
\text { coefficient }\end{array}$ & Std Error \\
\hline Age in years & $-0.133 * * *$ & $(0.021)$ & $-0.142 * * *$ & $(0.026)$ & $-0.137 * * *$ & $(0.031)$ \\
\hline Age in years ${ }^{2}$ & $0.001 * * *$ & $(1.40 \mathrm{E}-04)$ & $0.001 * * *$ & $(1.73 \mathrm{E}-04)$ & $0.001 * * *$ & $(2.07 \mathrm{E}-04)$ \\
\hline Female & $3.018 * * *$ & $(0.876)$ & $2.749 * *$ & $(1.082)$ & 2.066 & $(1.308)$ \\
\hline Age*Female & $-0.080 * * *$ & $(0.024)$ & $-0.066^{* *}$ & $(0.030)$ & -0.047 & $(0.035)$ \\
\hline $\operatorname{Age}^{2 *}$ Female & $0.001 * * *$ & $(1.67 \mathrm{E}-04)$ & $4.50 \mathrm{E}-04 * *$ & $(2.00 \mathrm{E}-04)$ & $3.18 \mathrm{E}-04$ & $(2.36 \mathrm{E}-04)$ \\
\hline \multicolumn{7}{|c|}{ Birth Cohort dummies: } \\
\hline AHEAD & $-0.123 * * *$ & $(0.040)$ & $-0.175 * * *$ & $(0.049)$ & $-0.210^{* * *}$ & $(0.059)$ \\
\hline CODA & $-0.126 * * *$ & $(0.040)$ & $-0.183 * * *$ & $(0.051)$ & $-0.197 * * *$ & $(0.061)$ \\
\hline HRS (ref.) & -- & & -- & & -- & \\
\hline WB & 0.042 & $(0.050)$ & 0.054 & $(0.065)$ & 0.100 & $(0.080)$ \\
\hline EBB & 0.053 & $(0.056)$ & 0.113 & $(0.073)$ & $0.186^{* *}$ & $(0.092)$ \\
\hline Constant & 1.040 & $(0.749)$ & 0.383 & $(0.958)$ & -0.514 & $(1.180)$ \\
\hline \# of subjects & 27,994 & & 27,994 & & 27,994 & \\
\hline \# of obs. & 145,802 & & 145,802 & & 145,802 & \\
\hline R-squared & $6.76 \%$ & & $7.44 \%$ & & $8.04 \%$ & \\
\hline Log likelihood & $-67,153$ & & $-46,738$ & & $-33,985$ & \\
\hline
\end{tabular}

$* \mathrm{p}<0.10, * * \mathrm{p}<0.05, * * * \mathrm{p}<0.01$.

Notes: $N=27,994$ subjects pooled from ten interview waves (ranging from 6,395 to 20,412 per wave). Logistic regressions with clustering for individual-level heteroskedasticity. No weights are applied. The observed means for one-plus, two-plus and three-plus disabilities are $19.5 \%, 11 \%$, and $7 \%$ respectively.

Source: Authors' calculations. 
Table 2: Effect of Number of ADL Disabilities on Risk of Nursing Home Admission, Cox Proportional Hazard Regression in a Competing Risk Model

\begin{tabular}{|c|c|c|c|c|}
\hline & \multicolumn{2}{|c|}{ Baseline \# ADL Disabilities } & \multicolumn{2}{|c|}{$\begin{array}{c}\text { Time-varying \# ADL } \\
\text { Disabilities }\end{array}$} \\
\hline & HR & $95 \% \mathrm{CI}$ & HR & $95 \%$ CI \\
\hline Female & $1.36 * * *$ & {$[1.20,1.54]$} & $1.29 * * *$ & {$[1.13,1.47]$} \\
\hline Age & $1.14 * * *$ & {$[1.13,1.15]$} & $1.13 * * *$ & {$[1.12,1.13]$} \\
\hline \multicolumn{5}{|c|}{ Number of ADL disabilities: } \\
\hline 0 (ref.) & -- & & -- & \\
\hline 1 & $1.95 * * *$ & {$[1.62,2.34]$} & $2.28 * * *$ & {$[1.93,2.70]$} \\
\hline 2 & $2.38 * * *$ & {$[1.85,3.06]$} & $3.33 * * *$ & {$[2.73,4.06]$} \\
\hline 3 & $3.17 * * *$ & {$[2.41,4.16]$} & $4.27 * * *$ & {$[3.40,5.37]$} \\
\hline 4 & $3.30 * * *$ & {$[2.20,4.94]$} & $3.97 * * *$ & {$[2.94,5.37]$} \\
\hline 5 & $3.19 * * *$ & {$[2.02,5.05]$} & $7.78 * * *$ & {$[5.89,10.27]$} \\
\hline 6 & $4.29 * * *$ & {$[2.60,7.08]$} & $4.30 * * *$ & {$[3.01,6.15]$} \\
\hline Weighted \# subjects & $64,440,000$ & & $64,440,000$ & \\
\hline Adjusted $\mathrm{R}^{2}$ & $75.9 \%$ & & $78.2 \%$ & \\
\hline Chi-square & 2,174 & & 2,529 & \\
\hline Df & 8 & & 8 & \\
\hline
\end{tabular}

$* \mathrm{p}<0.10,{ }^{* *} \mathrm{p}<0.05,{ }^{* * *} \mathrm{p}<0.01$.

Notes: $N=19,952$ respondents pooled from the AHEAD, CODA, HRS, WB, EBB cohorts in the Health and Retirement Survey over waves 1998 - 2010. The ADL disabilities assessed are difficulty bathing, dressing, eating, walking across a room, toileting, and transferring to/from a bed. There is complete information on disabilities (valid responses to all six ADL questions) for all respondents at baseline, but not in subsequent interview waves. Specifically, $5.7 \%$ of the time-varying observations on disabilities (unweighted) were incomplete. The regression using the time-varying number of disabilities includes a flag for such missing values (not significant).

Source: Authors' calculations. 
Table 3: Effect of Types of ADL Disabilities on Risk of Nursing Home Admission, Cox Proportional Hazard Regression in a Competing Risk Model

\begin{tabular}{|c|c|c|c|c|c|c|}
\hline \multirow[b]{3}{*}{ Variable } & \multirow{2}{*}{\multicolumn{2}{|c|}{$\begin{array}{l}\text { (1) } \\
\mathrm{omm} \rightarrow \mathrm{NH})\end{array}$}} & \multirow{2}{*}{\multicolumn{2}{|c|}{$\begin{array}{l}\text { (2) } \\
\mathrm{mm} \rightarrow \text { Death) }\end{array}$}} & \multirow{2}{*}{\multicolumn{2}{|c|}{$\begin{array}{c}\text { (3) } \\
h_{D}^{N}(\mathrm{NH} \rightarrow \text { Death })\end{array}$}} \\
\hline & & & & & & \\
\hline & HR & $95 \%$ CI & HR & $95 \% \mathrm{CI}$ & HR & $95 \% \mathrm{CI}$ \\
\hline Female & 1.14 & {$[0.97,1.34]$} & $0.65 * * *$ & {$[0.60,0.69]$} & $0.71 * * *$ & {$[0.61,0.83]$} \\
\hline Age & $1.11 * * *$ & {$[1.10,1.12]$} & $1.07 * * *$ & {$[1.07,1.08]$} & $1.04 * * *$ & {$[1.03,1.05]$} \\
\hline \multicolumn{7}{|l|}{ Types of ADL disability: } \\
\hline Dressing & 0.98 & {$[0.80,1.21]$} & $1.12 * *$ & {$[1.02,1.23]$} & 0.97 & {$[0.78,1.20]$} \\
\hline Walking across a room & $1.25 * *$ & {$[1.01,1.54]$} & $1.51 * * *$ & {$[1.37,1.67]$} & 1.12 & {$[0.91,1.38]$} \\
\hline Bathing/ Showering & $1.82 * * *$ & {$[1.48,2.24]$} & $1.64 * * *$ & {$[1.48,1.81]$} & $1.31 * *$ & {$[1.02,1.69]$} \\
\hline Eating & $1.25^{*}$ & {$[0.99,1.57]$} & $1.50 * * *$ & {$[1.34,1.67]$} & $1.16^{*}$ & {$[0.99,1.37]$} \\
\hline $\begin{array}{l}\text { Transferring to/from a } \\
\text { bed }\end{array}$ & 1.16 & {$[0.93,1.46]$} & 0.97 & {$[0.87,1.09]$} & 1.16 & {$[0.92,1.46]$} \\
\hline Toileting & 1.12 & {$[0.90,1.39]$} & 1.06 & {$[0.95,1.18]$} & 1.14 & {$[0.92,1.41]$} \\
\hline Nonwhite & $0.56 * * *$ & {$[0.46,0.68]$} & 0.94 & {$[0.86,1.02]$} & 1.09 & {$[0.89,1.33]$} \\
\hline Married & $0.55 * * *$ & {$[0.46,0.64]$} & $0.81 * * *$ & {$[0.75,0.86]$} & 0.93 & {$[0.77,1.14]$} \\
\hline Have LTC insurance & 1.00 & {$[0.81,1.23]$} & $0.85 * * *$ & {$[0.77,0.94]$} & 0.95 & {$[0.70,1.29]$} \\
\hline Cognition score & $0.59 * * *$ & {$[0.56,0.63]$} & $0.81 * * *$ & {$[0.79,0.84]$} & $0.87 * *$ & {$[0.79,0.97]$} \\
\hline \multicolumn{7}{|l|}{ Ever-have Disease history: } \\
\hline High blood pressure & 1.03 & {$[0.90,1.18]$} & $1.16^{* * *}$ & {$[1.09,1.24]$} & 1.03 & {$[0.88,1.21]$} \\
\hline Diabetes & $1.44 * * *$ & {$[1.24,1.67]$} & $1.51 * * *$ & {$[1.40,1.62]$} & $1.27 * * *$ & {$[1.08,1.49]$} \\
\hline Cancer & 0.96 & {$[0.81,1.15]$} & $1.82 * * *$ & {$[1.69,1.96]$} & $1.43 * * *$ & {$[1.21,1.70]$} \\
\hline Chronic lung disease & 1.18 & {$[0.96,1.44]$} & $2.04 * * *$ & {$[1.88,2.22]$} & $1.36 * * *$ & {$[1.13,1.64]$} \\
\hline Heart disease / attack & 1.06 & {$[0.93,1.21]$} & $1.52 * * *$ & {$[1.43,1.62]$} & $1.43 * * *$ & {$[1.24,1.65]$} \\
\hline Stroke & $1.44 * * *$ & {$[1.22,1.70]$} & $1.19 * * *$ & {$[1.10,1.30]$} & $0.85 * *$ & {$[0.74,0.99]$} \\
\hline Major psychiatric & $1.46 * * *$ & {$[1.24,1.72]$} & $1.21 * * *$ & {$[1.11,1.32]$} & 0.95 & {$[0.81,1.10]$} \\
\hline Arthritis & 0.90 & {$[0.78,1.03]$} & $0.93 * *$ & {$[0.87,0.99]$} & 0.93 & {$[0.80,1.09]$} \\
\hline Weighted \# subjects & \multicolumn{2}{|c|}{$64,440,000$} & \multicolumn{2}{|c|}{$66,920,000$} & \multicolumn{2}{|c|}{$3,670,000$} \\
\hline Adjusted $\mathbf{R}^{2}$ & \multicolumn{2}{|c|}{$84.8 \%$} & \multicolumn{2}{|c|}{$64.3 \%$} & \multicolumn{2}{|c|}{$14.0 \%$} \\
\hline Chi-squared & \multicolumn{2}{|l|}{30,464} & \multicolumn{2}{|l|}{7,080} & \multicolumn{2}{|l|}{219} \\
\hline Df & \multicolumn{2}{|l|}{32} & \multicolumn{2}{|l|}{32} & \multicolumn{2}{|l|}{30} \\
\hline
\end{tabular}

$* \mathrm{p}<0.10,{ }^{* *} \mathrm{p}<0.05,{ }^{* * *} \mathrm{p}<0.01$.

Notes: $N=19,952$ respondents pooled from the AHEAD, CODA, HRS, WB, EBB cohorts in the Health and Retirement Survey over waves 1998 - 2010. All covariates are time-varying except for sex. The cognition score is a scaled variable ranging from 0-7. In Column (1), the flags for missings for nonwhite, married, ever-have lung disease, arthritis, and cognition included are significant. The flags for missings for long-term care insurance, and the rest of the ever-have chronic conditions included in the regressions are not significant. In Column (2), the flags for missings for nonwhite, married, ever-have stroke, and cognition included are significant. The flags for missings for long-term care insurance, and the rest of the ever-have chronic conditions included in the regressions are not significant. In Column (3), only the flag for missings for cognition included is significant. The flags for missings for long-term care insurance, and all the ever-have chronic conditions included in the regressions are not significant. In all three regressions, the flags for missings for the ADL variables are omitted in the results due to too small proportions of missings.

Source: Authors' calculations. 
Table 4: Effects of Selected Covariates on Risk of Having Bathing Disability, Logistic Regression Model Estimation

\begin{tabular}{|c|c|c|}
\hline & Estimated coefficient & Standard Error \\
\hline Age in years & $0.032 * * *$ & $(0.012)$ \\
\hline Age in years ${ }^{2}$ & $0.001 * * *$ & $(2.36 \mathrm{E}-04)$ \\
\hline Female & 0.153 & $(0.157)$ \\
\hline Age*Female & 0.004 & $(0.014)$ \\
\hline Age $^{2} *$ Female & $0.63 \mathrm{E}-04$ & $(2.72 \mathrm{E}-04)$ \\
\hline Nonwhite & $0.176^{* * *}$ & $(0.041)$ \\
\hline Married & $-0.347 * * *$ & $(0.035)$ \\
\hline Have LTC insurance & $-0.162 * * *$ & $(0.053)$ \\
\hline Cognition score & $-0.206^{* * *}$ & $(0.017)$ \\
\hline \multicolumn{3}{|l|}{ Ever-have Disease history: } \\
\hline High blood pressure & -0.048 & $(0.034)$ \\
\hline Diabetes & $0.238 * * *$ & $(0.036)$ \\
\hline Cancer & -0.015 & $(0.042)$ \\
\hline Chronic lung disease & $0.351 * * *$ & $(0.044)$ \\
\hline Heart disease / attack & 0.012 & $(0.034)$ \\
\hline Stroke & $0.859 * * *$ & $(0.039)$ \\
\hline Major psychiatric & $0.586^{* * *}$ & $(0.037)$ \\
\hline Arthritis & $0.480 * * *$ & $(0.035)$ \\
\hline Currently smoke & $-0.095^{*}$ & $(0.050)$ \\
\hline Ever smoke & $0.128 * * *$ & $(0.037)$ \\
\hline Ever drink & $-0.441 * * *$ & $(0.034)$ \\
\hline \multicolumn{3}{|l|}{ Self-reported health: } \\
\hline Excellent & $-0.974 * * *$ & $(0.081)$ \\
\hline Very good & $-0.639 * * *$ & $(0.044)$ \\
\hline Good (ref.) & -- & -- \\
\hline Fair & $0.814^{* * *}$ & $(0.033)$ \\
\hline Poor & $1.807 * * *$ & $(0.039)$ \\
\hline Constant & $-4.341 * * *$ & $(0.159)$ \\
\hline \# of subjects & 27,994 & \\
\hline \# of obs. & 145,802 & \\
\hline $\mathrm{R}^{2}$ & $32.8 \%$ & \\
\hline Log likelihood & $-29,000$ & \\
\hline
\end{tabular}

$* \mathrm{p}<0.10, * * \mathrm{p}<0.05, * * * \mathrm{p}<0.01$.

Notes: $N=27,994$ subjects pooled from ten interview waves (ranging from 6,395 to 20,412 per wave). Logistic regression with clustering for individual-level heteroskedasticity. No weights are applied. The flags for missings for cognition, stroke, self-reported health included are significant. The flags for missings for nonwhite, married, longterm care insurance, smoking, drinking, and the rest of the ever-have chronic conditions included in the regressions are not significant.

Source: Authors' calculations. 


\section{Appendix 1: Descriptive Statistics}

Descriptive statistics are for covariates defined at 1998 baseline and used in the competing risk hazard regression analysis. Means with standard deviations are shown for all variables. Percentages are shown for dichotomous variables. $N=19,952$ respondents.

\begin{tabular}{|c|c|c|}
\hline & Mean & Std. Dev. \\
\hline Female & $57 \%$ & 0.50 \\
\hline Nonwhite & $17 \%$ & 0.38 \\
\hline \multicolumn{3}{|l|}{ As at wave 1998: } \\
\hline Baseline Age & 66.96 & 10.12 \\
\hline Walking ADL disability & $6.4 \%$ & 0.24 \\
\hline Dressing ADL disability & $9.4 \%$ & 0.29 \\
\hline Bathing ADL disability & $7.1 \%$ & 0.26 \\
\hline Eating ADL disability & $3.2 \%$ & 0.17 \\
\hline Transferring ADL disability & $6.5 \%$ & 0.25 \\
\hline Toileting ADL disability & $5.5 \%$ & 0.23 \\
\hline Married & $65.5 \%$ & 0.48 \\
\hline Have LTC insurance & $9.1 \%$ & 0.29 \\
\hline Currently smoke & $16.5 \%$ & 0.37 \\
\hline Ever smoke & $59.0 \%$ & 0.49 \\
\hline Ever drink & $47.4 \%$ & 0.50 \\
\hline Cognition score (scale of 1-7) & 4.43 & 0.84 \\
\hline \multicolumn{3}{|l|}{ Ever-have Disease history: } \\
\hline High blood pressure & $44.0 \%$ & 0.50 \\
\hline Diabetes & $13.4 \%$ & 0.34 \\
\hline Cancer & $10.3 \%$ & 0.30 \\
\hline Chronic lung disease & $7.2 \%$ & 0.26 \\
\hline Heart disease / attack & $20.6 \%$ & 0.40 \\
\hline Stroke & $6.8 \%$ & 0.25 \\
\hline Major psychiatric & $9.9 \%$ & 0.30 \\
\hline Arthritis & $49.4 \%$ & 0.50 \\
\hline \multicolumn{3}{|l|}{ Self-reported health: } \\
\hline Excellent & $12 \%$ & \\
\hline Very good & $26 \%$ & \\
\hline Good & $31 \%$ & \\
\hline Fair & $21 \%$ & \\
\hline Poor & $11 \%$ & \\
\hline \multicolumn{3}{|c|}{ Percentage by the Number of ADLs: } \\
\hline 0 & $83.1 \%$ & \\
\hline 1 & $7.7 \%$ & \\
\hline 2 & $3.6 \%$ & \\
\hline 3 & $2.2 \%$ & \\
\hline 4 & $1.3 \%$ & \\
\hline 5 & $1.1 \%$ & \\
\hline 6 & $1.0 \%$ & \\
\hline
\end{tabular}

